\title{
Higgs Strahlung at the Large Hadron Collider in the 2-Higgs-doublet model
}

\author{
Robert V. Harlander, Stefan Liebler and Tom Zirke \\ Fachbereich C, Bergische Universität Wuppertal, \\ 42097 Wuppertal, Germany \\ E-mail: harlander@physik.uni-wuppertal.de, liebler@uni-wuppertal.de, \\ t.zirke@uni-wuppertal.de
}

ABSTRACT: We present a calculation of all relevant contributions to associated production of a Higgs boson with a weak gauge boson in the 2-Higgs-doublet model (2HDM) at the LHC, $p p \rightarrow \phi$, with $\phi \in\left\{h, H^{0}, A\right\}$ and $V \in\{W, Z\}$. While for the $W \phi$ mode, this mostly amounts to a simple rescaling of the Standard Model (SM) cross section, the $Z \phi$ cross section depends on several $2 \mathrm{HDM}$ parameters. The ratio $\sigma^{W \phi} / \sigma^{Z \phi}$, for which we present the currently most complete SM prediction, therefore appears to be a sensitive probe of possible New Physics effects. We study its numerical dependence on the top and bottom Yukawa couplings, including their sign. Furthermore, we consider the $W \phi / Z \phi$ ratio in exemplary $2 \mathrm{HDM}$ scenarios and briefly address the effects in the boosted regime. Analogous studies for other 2HDM scenarios will become possible with an upcoming version of the program vh@nnlo which incorporates the 2HDM effects.

KeYwords: QCD Phenomenology, Hadronic Colliders

ARXIV EPRINT: 1307.8122 


\section{Contents}

1 Introduction $\quad 1$

2 Theoretical description of the Higgs Strahlung process 3

2.1 Standard Model 3

2.2 2-Higgs-doublet model 4

2.3 Implementation in vh@nnlo $\quad 7$

2.4 Input parameters 8

2.5 The $W \phi / Z \phi$ ratio 8

3 Effect of the Yukawa couplings $\quad 9$

4 2-Higgs-doublet model $\quad 12$

$\begin{array}{ll}4.1 \text { Choice of 2HDM parameters } & 12\end{array}$

$4.2 g g Z \phi$ and $b \bar{b} Z \phi$ contributions involving internal (pseudo)scalars $\quad 12$

4.3 Light Higgs 13

$\begin{array}{ll}4.4 \text { Heavy and pseudoscalar Higgs } & 18\end{array}$

5 Effects in the boosted regime $\quad 19$

$\begin{array}{ll}\text { 5.1 The gluon-induced component in the SM } & 19\end{array}$

5.2 Modified SM Yukawa couplings 21

$\begin{array}{lll}5.3 & \text { 2-Higgs-doublet model } & 22\end{array}$

6 Conclusions $\quad 24$

$\begin{array}{ll}\text { A 2-Higgs-doublet model } & 25\end{array}$

\section{Introduction}

After the discovery of a Higgs boson at the Large Hadron Collider (LHC) [1, 2], one of the top priorities of the experiments is to measure its properties as precisely as possible. Any deviation from the Standard Model (SM) predictions could be valuable information about a possible extended theory. ${ }^{1}$

However, precision physics at hadron colliders like the LHC typically suffers from large experimental and theoretical uncertainties. The latter are dominated by the truncation of the perturbative series and insufficient knowledge of the input parameters such as the strong coupling and the parton density functions. Observables where these uncertainties are absent or suppressed are therefore highly desirable.

\footnotetext{
${ }^{1}$ For a recent review of theory predictions for Higgs physics at the LHC, see ref. [3].
} 
In this paper, we consider the Higgs Strahlung process, where a Higgs boson is produced in association with a $W$ or a $Z$ boson. In the region below about $m_{H}=125 \mathrm{GeV}$, this was the dominant search mode at the Tevatron, with the Higgs decaying into $b \bar{b}$ and the gauge boson decaying leptonically [4]. The data show an excess above the expected background which is, however, compatible with a background fluctuation. At the LHC, ATLAS has searched for a signal from Higgs Strahlung in the $H \rightarrow W W^{\star}[5], H \rightarrow b \bar{b}[6]$, and $H \rightarrow$ invisible modes [7], providing an upper limit on the cross section of 1.4 times the SM expectation for $m_{H}=125 \mathrm{GeV}$ (driven by the $H \rightarrow b \bar{b}$ search). CMS observes a $2.1 \sigma$ excess at $m_{H}=125 \mathrm{GeV}$ in the $V H \rightarrow V b \bar{b}$ mode which is compatible with the SM expectation [8]. Searches for $H \rightarrow$ invisible [9] and $H \rightarrow W W^{(*)}$ [10] from Higgs Strahlung are compatible with the background; the latter provides an upper limit on the cross section of around ten times the SM expectation. In summary, there are first hints of the Higgs Strahlung process, but presumably it will only be after the current shut-down of the LHC when this process can be firmly established.

The theory prediction for its total inclusive cross section within the framework of the SM is known through next-to-next-to-leading order (NNLO) QCD $[11,12]$ and NLO electroweak (EW) corrections [13]. Soft gluon resummation effects are negligibly small compared to the fixed order NNLO result [14], indicating an excellent convergence of the perturbative series. Fully differential predictions for Higgs Strahlung at NNLO QCD [15] and including the NLO electro-weak effects [16] are also available (see also ref. $[3,17]$ ). Through NLO QCD, the ratio of the cross sections for the $W H$ and the $Z H$ process is practically free from QCD corrections. Clearly, this ratio is quite insensitive to uncertainties due to the parton density functions (PDFs) as well, since both processes have very similar initial states.

At NNLO, however, the ratio of the $W H$ to $Z H$ rate depends on the top and bottom quark Yukawa couplings, due to additional contributions specific to $Z H$ production. They are mostly due to gluon initiated processes $[12,18,19]$ which involve closed third-generation quark loops. The $W H / Z H$ ratio should thus be a useful quantity to study at the LHC, providing direct access to some of the most crucial parameters of the Higgs Lagrangian.

The 2-Higgs-doublet model (2HDM) is one of the simplest extensions of the SM Higgs sector (for reviews, see refs. [20-25]). Recently, a number of studies have been performed in order to scan the parameter space of a $2 \mathrm{HDM}$ for regions which are still allowed in the light of the existence of a Higgs boson with $m_{H}=125 \mathrm{GeV}$ [25-42]. In this paper, we assume $\mathrm{CP}$ conservation in the Higgs sector and no tree-level flavor-changing neutral currents. The spectrum of neutral physical Higgs bosons of the 2HDM then consists of two CP-even Higgs bosons $h$ and $H^{0}$ (where, by definition, $m_{h}<m_{H^{0}}$ ), and one CP-odd Higgs boson $A$, often referred to as the pseudoscalar Higgs. According to the structure of the Yukawa couplings, one distinguishes four types of $2 \mathrm{HDMs}$, only two of which lead to different results in the following (see appendix A).

A short review of Higgs Strahlung within the 2HDM can be found in ref. [43]. As it is well-known, a simple reweighting of the SM Higgs Strahlung cross sections according to the different couplings of the Higgs bosons $\phi \in\left\{h, H^{0}, A\right\}$ to the gauge bosons $V \in\{W, Z\}$ is not sufficient. This is due to higher order contributions which involve top and bottom 
Yukawa couplings. In this paper, we thoroughly combine all contributions to the Higgs Strahlung cross section, taking into account $2 \mathrm{HDM}$ effects. We will show that modified top and bottom Yukawa couplings mostly affect the $Z \phi$ production cross section and thus can lead to significant deviations of the ratio of the $W \phi / Z \phi$ cross sections with respect to its SM value. In addition, the final state $Z \phi$ can also be produced via $s$-channel exchange of a (virtual) scalar $\phi^{\prime} \neq \phi$ in the $2 \mathrm{HDM}$ which is produced through either $b \bar{b}$ or $g g$ annihilation. This can enhance the $Z \phi$ production cross section dramatically. Such effects have been studied for $Z A$ production in refs. [44-50] and for $Z h$ and $Z H^{0}$ production in ref. [50]. Most of these studies were carried out in the framework of the Minimal Supersymmetric SM (MSSM) though, where the parameters of the Higgs sector are tightly constraint, thus restricting the possible effects with respect to a fully general 2HDM. For our numerical analysis, we extended the program vh@nnlo $[12,51]$ for the calculation of associated $V H$ production to $V \phi$ production in the $2 \mathrm{HDM}$.

While the main results of the paper are derived from predictions for the total inclusive cross section, we also study the influence of lower cuts on the Higgs' transverse momentum $p_{T}^{\phi}$, motivated by the increased signal-to-background ratio in the so-called boosted regime [52]. We will argue that, while the sensitivity to the Yukawa couplings is generally increased in that case, effects induced by additional (virtual) scalar particles can be strongly reduced. This suggests that events at low- $p_{T}^{\phi}$ should not be discarded in experimental analyses.

The remainder of the paper is structured as follows: section 2 reviews Higgs Strahlung in the SM, before discussing the various cross section contributions in the 2HDM. Their implementation within vh@nnlo is described, and the $W \phi$ to $Z \phi$ ratio of production cross sections as precision observable is motivated. We provide the most up-to-date numerical value for this ratio in the SM. In section 3, the effect of Yukawa couplings on Higgs Strahlung in the SM is discussed. Section 4 describes different aspects of Higgs Strahlung in the 2HDM and highlights their effects on the $W \phi$ to $Z \phi$ ratio. Differences of the observed effects in the boosted regime are discussed in section 5 . Conclusions and an outlook for possible future studies are given in section 6. Appendix A summarizes some information on the 2HDM which is relevant for our presentation.

\section{Theoretical description of the Higgs Strahlung process}

\subsection{Standard Model}

The cross section for Higgs Strahlung in the SM through NNLO QCD can be written as

$$
\sigma^{V H}=\sigma_{\mathrm{DY}}^{V H}+\sigma_{\mathrm{I}}^{V H}+\delta_{V Z} \cdot\left(\sigma_{\mathrm{II}}^{Z H}+\sigma_{g g Z H}\right)
$$

Through NLO QCD, i.e. $\mathcal{O}\left(\alpha_{s}\right)$, only the so-called "Drell-Yan-like" (DY) terms $\sigma_{\mathrm{DY}}^{V H}$ contribute. They are given by the production of a virtual gauge boson $V^{*}$ and its subsequent decay into a real gauge boson plus a Higgs; the leading order diagram is shown in figure 1 (a). We exclude contributions from the definition of $\sigma_{\mathrm{DY}}^{V H}$ which involve the coupling of the gauge boson to a closed quark loop, see for example figure $1(b)$, which will be 
attributed to $\sigma_{g g Z H}$. The QCD corrections to $\sigma_{\mathrm{DY}}^{V H}$ are therefore simply given by the QCD corrections to the Drell-Yan process $q \bar{q} \rightarrow V^{*}$, and they are the same for $W H$ and $Z H$ production at any order of perturbation theory. At NNLO, they have been obtained in ref. [12] on the basis of ref. [53].

The other terms of eq. (2.1) only contribute at $\mathcal{O}\left(\alpha_{s}^{2}\right)$ or higher. They all involve top- or bottom-quark loops. The term $\sigma_{\mathrm{I}}^{V H}$ is very similar for $W H$ and $Z H$ production. Its Feynman diagrams can be obtained by inserting a top- or bottom-quark loop into the gluon lines of (real and virtual) NLO QCD diagrams for $p p \rightarrow V$ production, and radiating the Higgs boson off this loop. A sample diagram is shown in figure 2 (a); it is their interference with DY-diagrams which leads to $\sigma_{\mathrm{I}}^{V H}$. The numerical effect of this contribution was evaluated in the heavy-top limit and by neglecting the bottom Yukawa coupling; it was found to be of the order of $1-2 \%$ of the total cross section [54].

The terms $\sigma_{\mathrm{II}}^{Z H}$ and $\sigma_{g g Z H}$ collect contributions where the $Z$ boson couples to a closed top- or bottom-quark loop. The analogous terms are absent for $W H$ production as indicated by the Kronecker symbol $\delta_{V Z}$ in eq. (2.1). The Higgs boson may then be emitted either from the $Z$ boson, or from the closed quark loop. In $\sigma_{\mathrm{II}}^{Z H}$, the quark loop is connected via gluons to an external quark line. This results in either two-loop diagrams for $q \bar{q} \rightarrow Z H$, see figure 2 (b), or in one-loop diagrams with two quarks, one gluon, and $Z H$ as external states. The resulting amplitudes have to be interfered with the corresponding lowest order DY-contribution. In ref. [54], $\sigma_{\mathrm{II}}^{Z H}$ was found to be even smaller than $\sigma_{\mathrm{I}}^{V H}$, typically at the sub-percent level.

Of particular importance for our analysis is the contribution $\sigma_{g g Z H}$, where the closed top- or bottom-quark loop is connected to two initial state gluons, the $Z$ is radiated off that loop, and the Higgs is emitted from the $Z$ or from the quark loop. The lowest order, i.e. $\mathcal{O}\left(\alpha_{s}^{2}\right)$, was calculated in refs. $[12,18,19]$. Due to the two initial state gluons, its numerical contribution to the total cross section can be significantly larger than the one of $\sigma_{\text {II }}^{Z H}$, although both are formally of the same order of perturbation theory. Its size depends on the center-of-mass energy though: while it was negligibly small for the Tevatron, for example, it amounts to about $4 \%(6 \%)$ at the $\mathrm{LHC}$ for $8 \mathrm{TeV}(14 \mathrm{TeV})$. Since its impact on the theoretical uncertainty of the total cross section was quite significant, ref. [55] evaluated it at the next order in perturbation theory, i.e. $\mathcal{O}\left(\alpha_{s}^{3}\right)$, which is formally an $\mathrm{N}^{3} \mathrm{LO}$ contribution to the Higgs Strahlung process. These QCD corrections where found to be of the order of $100 \%$, similar to what is observed in the gluon fusion process $g g \rightarrow H$ [5662]. While the absolute renormalization scale dependence is almost the same at $\mathcal{O}\left(\alpha_{s}^{2}\right)$ and $\mathcal{O}\left(\alpha_{s}^{3}\right)$, the relative variation is smaller at $\mathcal{O}\left(\alpha_{s}^{3}\right)$ by a factor of two due to the large K-factor.

\subsection{2-Higgs-doublet model}

In the SM, contributions proportional to the bottom Yukawa coupling are typically strongly suppressed with respect to the corresponding top-quark induced terms. This is different in extended Higgs sectors like, for example, the 2HDM. An introduction to the 2HDM including a presentation of all relevant couplings in the neutral Higgs sector is given in appendix A. In this paper, we consider the production of any of the three neutral Higgs bosons of the 


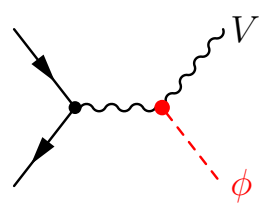

(a)

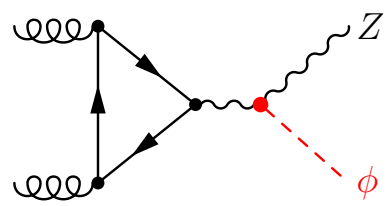

(b)

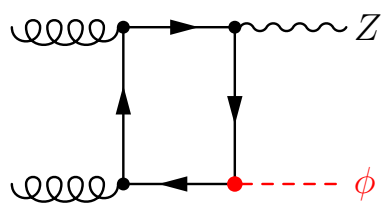

(c)

Figure 1. Leading order Feynman diagrams: (a) Drell-Yan-like contribution $\sigma_{\mathrm{DY}}^{V \phi}$; (b,c) gluoninitiated contributions $\sigma_{g g Z \phi}$. Couplings which differ between SM and 2HDM are highlighted.

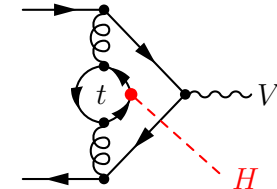

(a)

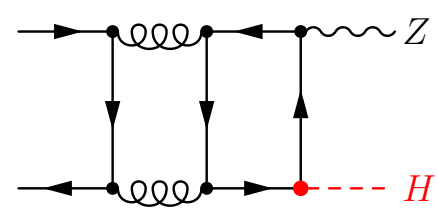

(b)

Figure 2. Contribution to (a) $\sigma_{\mathrm{I}}^{V H}$ and (b) $\sigma_{\mathrm{II}}^{Z H}$.

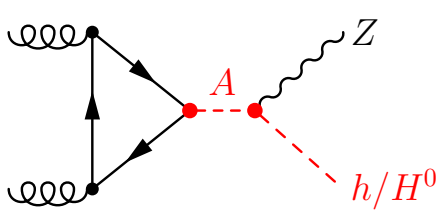

(a)

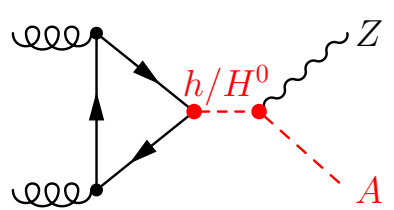

(b)

Figure 3. Additional contributions to gluon-initiated Higgs Strahlung in case of the 2HDM.

2HDM, generically denoting them as $\phi \in\left\{h, H^{0}, A\right\}$, in association with a weak gauge boson $V \in\{W, Z\}$. The main differences to the SM are as follows:

- The couplings $g_{V V}^{\phi}$ are different from the SM $g_{V V}^{H}$ coupling.

- The bottom Yukawa coupling can be significantly enhanced and thus cannot be neglected in general.

- In addition to an off-shell gauge boson, also one of the three neutral Higgs bosons can occur as internal particle.

The implications of these changes are as follows. The DY contribution $\sigma_{\mathrm{DY}}^{V \phi}$ is obtained from the SM expression simply by reweighting the Higgs coupling to the gauge bosons:

$$
\sigma_{\mathrm{DY}}^{V \phi}=\left(g_{V V}^{\phi}\right)^{2} \cdot \sigma_{\mathrm{DY}}^{V H} .
$$

The 2HDM version of the gluon-initiated process, $\sigma_{g g Z \phi}$, on the other hand, depends on $g_{Z Z}^{\phi}$ as well as on the relative Yukawa couplings $g_{b}^{\phi}$ and $g_{t}^{\phi}$. In addition, instead of the intermediate $Z$ boson in figure 1 (b), a (virtual) CP-odd/even Higgs boson $\phi^{\prime}$ can be produced, which decays into a real CP-even/odd Higgs boson and a $Z$ boson, see figure $3[44-46,48-50]$. As we will see, this contribution is absolutely essential for the correct 


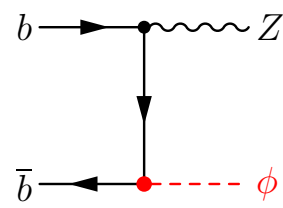

(a)

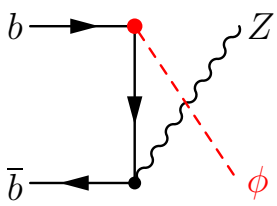

(b)

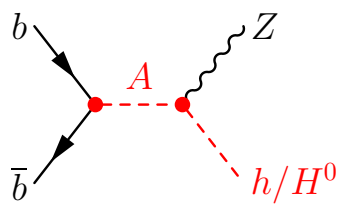

(c)

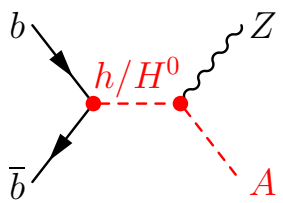

(d)

Figure 4. $b \bar{b} Z \phi$ contributions to Higgs Strahlung in the 2HDM.

description of the Higgs Strahlung process in the 2HDM. This is particularly true if the mass of the intermediate particle $\phi^{\prime}$ is larger than the threshold, $m_{\phi^{\prime}}>m_{Z}+m_{\phi}$, in which case the propagator becomes resonant at the partonic cms energy $\sqrt{\hat{s}}=m_{\phi^{\prime}}$. We regulate the associated divergence by replacing the propagator as

$$
\frac{1}{\hat{s}-m_{\phi^{\prime}}^{2}} \rightarrow \frac{1}{\hat{s}-m_{\phi^{\prime}}^{2}+i m_{\phi^{\prime}} \Gamma_{\phi^{\prime}}},
$$

where $\Gamma_{\phi^{\prime}}$ is the total width of $\phi^{\prime}$. Given a specific $2 \mathrm{HDM}, \Gamma_{\phi^{\prime}}$ is calculable; we use the value provided by 2 HDMC $[63,64]$, which can be linked to the new version of vh@nnlo.

Since the $\mathcal{O}\left(\alpha_{s}^{3}\right)$ effects for $\sigma_{g g Z \phi}$ are only known for vanishing bottom Yukawa coupling [55], we will disregard these corrections in the 2HDM analysis and take into account only the $\mathcal{O}\left(\alpha_{s}^{2}\right)$ results $[12,18,19]$.

For the 2HDM generalizations $\sigma_{\mathrm{I}}^{V \phi}$ and $\sigma_{\mathrm{II}}^{Z \phi}$ of $\sigma_{\mathrm{I}}^{V H}$ and $\sigma_{\mathrm{II}}^{Z H}$, also Feynman diagrams with closed bottom instead of top quark loops should be taken into account. Due to the fact that one cannot evaluate them in an effective theory analogous to the heavy-top limit, these contributions are extremely difficult to calculate and thus currently unavailable. Considering the smallness of these terms in the SM, however, it should be fair to neglect these terms altogether. Unless stated otherwise, we therefore set $\sigma_{\mathrm{I}}^{V \phi}=\sigma_{\mathrm{II}}^{Z \phi}=0$ in what follows.

Finally, an enhanced bottom Yukawa coupling induces a new contribution $\sigma_{b \bar{b} Z \phi} \sim\left(y_{b}^{\phi}\right)^{2}$ with respect to the SM, where the final state $Z \phi$ is produced in association with bottom quarks $[45,47-50]$. If the latter are not tagged, the cross section can be calculated in the socalled five-flavour scheme where the perturbative series is re-arranged to resum logarithmic terms that arise from the collinear region of the final state bottom quark momenta (see, e.g., ref. [65] and references therein). The bottom quarks then appear as initial states, $b \bar{b} \rightarrow Z \phi$, with proper parton density functions. The relevant Feynman diagrams at leading order are depicted in figure 4 . Similar to the process $g g Z \phi$, they partly involve intermediate virtual Higgs bosons $\phi^{\prime}$, which we treat in the very same way as described above (see eq. (2.3)).

For the purpose of the current paper, the LO prediction of the $b \bar{b} Z \phi$ process shall be sufficient. We will see, however, that this contribution can be quite significant and even numerically dominant, in particular if the intermediate Higgs boson becomes resonant. NLO corrections to this process have been calculated in ref. [49]. They should be included in the analysis once sufficient experimental data are available.

Note that $\sigma_{b \bar{b} Z \phi}$ exclusively refers to that part of the partonic process $b \bar{b} \rightarrow Z \phi$ which involves a bottom Yukawa coupling. The same process can also be mediated for $y_{b}=0$, 
namely as part of $\sigma_{\mathrm{DY}}^{Z \phi}$, which we evaluate with five-flavor PDFs. Assuming massless bottom quarks, there is no interference between these DY- and the $b \bar{b} Z \phi$-diagrams.

To summarize, we write the cross section for Higgs Strahlung in the 2HDM as

$$
\sigma^{V \phi}=\sigma_{\mathrm{DY}}^{V \phi}+\delta_{V Z} \cdot\left(\sigma_{g g Z \phi}+\sigma_{b \bar{b} Z \phi}\right) .
$$

\subsection{Implementation in vh@nnlo}

For the numerical evaluation of the individual contributions, we use the program vh@nnlo [51] which we extended to the $2 \mathrm{HDM}$. It turns out convenient to link vh@nnlo to 2HDMC $[63,64]$ to allow for different parametrizations of the 2HDM, consistency checks of the input parameters, and additional information about the decays of the involved scalar particles. The latest version of vh@nnlo includes:

- The Drell-Yan-like terms $\sigma_{\text {DY }}^{V \phi}$ through NNLO by integrating the results of zwprod [53] and re-weighting with the proper $2 \mathrm{HDM}$ coupling.

- The gluon-initiated terms $g g Z \phi$ at $\mathcal{O}\left(\alpha_{s}^{2}\right)$ for finite top and bottom Yukawa coupling, including the full top- and bottom-quark mass dependence, as well as terms with internal Higgs bosons, see figure 3. For the top- and bottom-quark masses, the on-shell values are used throughout the calculation of the $g g Z \phi$ contribution. The corresponding part of the original vh@nnlo has been fully replaced by an updated code which provides higher flexibility concerning the choice of physical parameters. It was generated with the help of FeynArts/FormCalc $[66,67]$ and requires vh@nnlo to be linked to the LoopTools [67] and CUBA [68] libraries. Regarding the resonant exchange of a Higgs boson $\phi^{\prime} \in\left\{h, H^{0}, A\right\}$ (see figure 3), we introduce the BreitWigner function as given in eq. (2.3) and insert the numerical value for the total width of $\phi^{\prime}$ provided by $2 \mathrm{HDMC}[63,64]$. The numerical integration of the BreitWigner peak is done by suitable Monte Carlo sampling.

- The bottom-quark initiated terms $b \bar{b} Z \phi$ at LO. The bottom mass entering the Yukawa coupling is taken to be the $\overline{\mathrm{MS}}$ mass at the energy scale $\sqrt{\hat{s}}$ of the incoming partons. The resonant contribution from internal (pseudo)scalars (see figures $4(\mathrm{c}, \mathrm{d})$ ) is treated in the very same way as for the $g g Z \phi$ terms, see above.

The only genuinely new terms with respect to the previous version of vh@nnlo are $b \bar{b} Z \phi$ and the $s$-channel contributions to $g g Z \phi$. Corresponding results have been reported on in the literature before [44-50]. We re-calculated them with the help of FeynArts/FormCalc [66, 67], employing an adapted version of the $2 \mathrm{HDM}$ model file. Our analytic $b \bar{b} Z \phi$ result agrees with the one of ref. [50]. Numerical comparison of our results with the literature is impeded by their rather crucial dependence on the precise value of the parameters of the Higgs sector. Since most of the previous analysis focus on the MSSM, such comparisons could only be done at a rather qualitative level.

For $b \bar{b} Z \phi\left(\phi \in\left\{h, H^{0}, A\right\}\right)$ and $g g Z A$ production, we find satisfactory numerical agreement with ref. [50], but we fail to reproduce the numbers for $g g \rightarrow Z h$ and $g g \rightarrow Z H^{0}$. However, while our results for $Z h$ production obey the required decoupling limit by approaching 
the SM result for large $M_{A}$, for example, we cannot verify this behavior in ref. [50]. The source of this difference is unresolved. ${ }^{2}$

In case of the SM, higher order terms as presented in section 2.1 are available and recommended to be included:

- Electro-weak contributions by assuming full factorization with the Drell-Yan-like terms, i.e. by replacing [69]

$$
\sigma_{\mathrm{DY}}^{V H} \rightarrow\left(1+\delta_{\mathrm{EW}}^{V H}\right) \sigma_{\mathrm{DY}}^{V H}
$$

The correction factor $\delta_{\mathrm{EW}}^{V H}$ is implemented by interpolating a table of numerical results obtained from refs. [13, 70].

- The terms $\sigma_{\mathrm{I}}^{V H}$ and $\sigma_{\mathrm{II}}^{Z H}$, by implementing the results of ref. [54].

- The gluon-initiated terms $g g Z H$ at $\mathcal{O}\left(\alpha_{s}^{3}\right)$ with vanishing bottom Yukawa coupling, by implementing the perturbative correction factor in the heavy-top approximation [55].

\subsection{Input parameters}

Throughout this paper, we use the following numerical values for the SM parameters:

$$
\begin{aligned}
m_{t}^{\text {pole }} & =172.3 \mathrm{GeV}, & m_{b}^{\text {pole }} & =4.75 \mathrm{GeV}, \quad m_{b}^{\overline{\mathrm{MS}}}\left(m_{b}\right)=4.16 \mathrm{GeV}, \\
m_{W} & =80.398 \mathrm{GeV}, & m_{Z} & =91.1876 \mathrm{GeV}, \\
\Gamma_{W} & =2.141 \mathrm{GeV}, & \Gamma_{Z} & =2.4952 \mathrm{GeV} \\
G_{F} & =1.16637 \cdot 10^{-5} \mathrm{GeV}^{-2}, & \sin ^{2} \theta_{C} & =0.0508
\end{aligned}
$$

where $\theta_{C}$ is the Cabbibo mixing angle (mixing with the third quark generation can be safely neglected); the notation for the other quantities should be self-explanatory. Hadronic cross sections are calculated for a cms energy of $\sqrt{s}=14 \mathrm{TeV}$. Furthermore, we use a value of $\alpha_{s}\left(m_{Z}\right)=0.119$ as input for 2HDMC. For the cross section calculations however, the strong coupling constant is taken according to the corresponding parton density functions. As default set we use MSTW2008NNLO [71], for which $\alpha_{s}\left(m_{Z}\right)=0.11707$.

\subsection{The $W \phi / Z \phi$ ratio}

From the previous discussion, it is clear that the ratio of the cross sections for $W \phi$ and $Z \phi$ production

$$
R_{W Z \phi}=\frac{\sigma^{W \phi}}{\sigma^{Z \phi}}
$$

is rather insensitive to radiative corrections in the SM. This feature is shared with the Drell-Yan production of lepton pairs, of course. However, due to the fact that $R_{W Z \phi}$ is sensitive to Higgs couplings to fermions, it provides a useful handle on possible deviations from the SM as soon as precise measurements of the ratio in eq. (2.7) are available. From

\footnotetext{
${ }^{2}$ Thanks to B. Kniehl for discussions on this issue.
} 
the experimental point of view, the similarity of the $W \phi$ and $Z \phi$ production processes should be reflected in a reduced uncertainty on the measured value of $R_{W Z \phi}$ as well. Any dependence on the beam luminosity drops out, for example; also $b$-tagging efficiencies are basically the same in the numerator and the denominator, with a marginal difference from kinematics due to $m_{W} \neq m_{Z}$.

The theoretical prediction for $R_{W Z H}$ within the SM is rather precise, since both the numerator and the denominator are known with NNLO accuracy. Using vh@nnlo, we calculate $\sigma_{\mathrm{DY}}^{V H}$ including electro-weak corrections, $\sigma_{g g Z H}$ at $\mathcal{O}\left(\alpha_{s}^{3}\right), \sigma_{\mathrm{I}}^{V H}, \sigma_{\mathrm{II}}^{Z H}$ and $\sigma_{b \bar{b} Z H}$, using the input parameters from section 2.4. When varying parameters for error estimation, we take the ratio at each point. We use the NNLO PDF sets from MSTW2008 [71], CT10 [72], and NNPDF23 [73], calculate the envelope of the error bands obtained from each set, and take its center and half width as prediction for $R_{W Z H}$ and its PDF $+\alpha_{S}$ uncertainty. For the estimation of the scale uncertainty, we vary the renormalization and factorization scale separately in an interval $\left[\frac{1}{3} \mu_{0}, 3 \mu_{0}\right]$ around our default choice $\mu_{0}=m_{V H}$, the invariant mass of the $V H$ system. The total uncertainty is obtained by adding both numbers linearly. Our results for various values of the Higgs mass are listed in table 1.

It is instructive to compare the uncertainty of the ratio $R_{W Z H}$ to those of the $V H$ cross sections. For $m_{H}=125 \mathrm{GeV}$ we obtain

$$
\begin{aligned}
\sigma_{\mathrm{SM}}^{W H} & =1.520 \mathrm{pb} \pm 0.6 \%^{\text {scale }} \pm 2.1 \%^{\mathrm{PDF}+\alpha_{S}}, \\
\sigma_{\mathrm{SM}}^{Z H} & =0.970 \mathrm{pb} \pm 3.2 \%^{\text {scale }} \pm 1.9 \%^{\mathrm{PDF}+\alpha_{S}}, \\
R_{W Z H}^{\mathrm{SM}} & =1.570 \pm 2.6 \%^{\text {scale }} \pm 0.7 \%^{\mathrm{PDF}+\alpha_{S}}
\end{aligned}
$$

An uncorrelated error estimate for the ratio, i.e. adding the relative uncertainties of $\sigma_{\mathrm{SM}}^{W H}$ and $\sigma_{\mathrm{SM}}^{Z H}$ in quadrature, would lead to about $5.8 \%$ in total compared to $3.4 \%$. Thus we observe that a significant part of the uncertainties indeed cancels in the ratio. The residual uncertainty is dominated by the scale uncertainty of the $g g Z H$ contribution to $\sigma^{Z H}$, which has no counterpart in $\sigma^{W H}$ to cancel against.

In the rest of this paper, we will study the influence of possible non-SM physics on the ratio $R_{W Z \phi}$ for the production of a neutral Higgs boson $\phi$. In a first step, we will simply modify the SM Yukawa couplings, without any particular underlying model in mind. Subsequently, we will study a general 2HDM, taking into account all the effects discussed in section 2.2 .

In the following discussion, we use eq. (2.4) as the definition of $\sigma^{V \phi}$, without $\mathcal{O}\left(\alpha_{s}^{3}\right)$ corrections to $\sigma_{g g Z H}$ and electro-weak effects, since they are only known in the SM.

\section{Effect of the Yukawa couplings}

In this section, we will not respect any constraints on the third generation Yukawa couplings of the SM, but simply test the sensitivity of the ratio $R_{W Z \phi}$ on these parameters. For that purpose, we rescale the Yukawa couplings as

$$
y_{t}=\kappa y_{t}^{\mathrm{SM}} \quad \text { and } / \text { or } \quad y_{b}=y_{b}^{\mathrm{SM}} / \kappa
$$

where $y_{t}^{\mathrm{SM}}$ and $y_{b}^{\mathrm{SM}}$ are the SM values of the top- and the bottom Yukawa couplings. 


\begin{tabular}{|c|c|c|c|c|}
\hline$m_{H}[\mathrm{GeV}]$ & $R_{W Z H}^{\mathrm{SM}}$ & $\Delta_{\text {scale }}[\%]$ & $\Delta_{\mathrm{PDF}+\alpha_{s}}[\%]$ & $\Delta_{\text {total }}[\%]$ \\
\hline 100 & 1.676 & \pm 1.9 & \pm 0.7 & \pm 2.6 \\
110 & 1.630 & \pm 2.3 & \pm 0.6 & \pm 2.9 \\
120 & 1.587 & \pm 2.4 & \pm 0.8 & \pm 3.3 \\
122 & 1.580 & \pm 2.6 & \pm 0.8 & \pm 3.3 \\
124 & 1.570 & \pm 2.7 & \pm 0.7 & \pm 3.3 \\
125 & 1.570 & \pm 2.6 & \pm 0.7 & \pm 3.4 \\
126 & 1.563 & \pm 2.7 & \pm 0.7 & \pm 3.4 \\
128 & 1.556 & \pm 2.8 & \pm 0.9 & \pm 3.7 \\
130 & 1.545 & \pm 2.8 & \pm 0.8 & \pm 3.6 \\
140 & 1.501 & \pm 3.1 & \pm 1.0 & \pm 4.1 \\
150 & 1.470 & \pm 3.4 & \pm 0.8 & \pm 4.1 \\
160 & 1.428 & \pm 3.6 & \pm 0.8 & \pm 4.5 \\
170 & 1.406 & \pm 3.7 & \pm 0.9 & \pm 4.6 \\
180 & 1.385 & \pm 3.9 & \pm 0.9 & \pm 4.7 \\
190 & 1.388 & \pm 4.0 & \pm 0.9 & \pm 5.0 \\
200 & 1.381 & \pm 4.1 & \pm 0.9 & \pm 5.0 \\
210 & 1.382 & \pm 3.9 & \pm 0.9 & \pm 4.9 \\
220 & 1.388 & \pm 3.8 & \pm 0.9 & \pm 4.7 \\
230 & 1.397 & \pm 3.6 & \pm 0.8 & \pm 4.5 \\
240 & 1.412 & \pm 3.3 & \pm 0.8 & \pm 4.2 \\
250 & 1.430 & \pm 3.1 & \pm 0.8 & \pm 3.9 \\
260 & 1.446 & \pm 2.9 & \pm 0.8 & \pm 3.6 \\
270 & 1.469 & \pm 2.6 & \pm 0.8 & \pm 3.3 \\
280 & 1.487 & \pm 2.4 & \pm 0.7 & \pm 3.0 \\
290 & 1.508 & \pm 2.1 & \pm 0.7 & \pm 2.8 \\
300 & 1.524 & \pm 1.9 & \pm 0.7 & \pm 2.5 \\
\hline & & & & \\
\hline
\end{tabular}

Table 1. Ratio $\sigma^{W H}$ over $\sigma^{Z H}$ in the SM for $\sqrt{s}=14 \mathrm{TeV}$ with error estimation from scale variation and PDF $+\alpha_{S}$ uncertainties. The numerical error induced by the Monte Carlo integration is expected to have an effect on the last digit only.

Figures $5(\mathrm{a}-\mathrm{c})$ show the various contributions to the total $Z H$ production cross section as discussed above. For $\kappa=1$, all figures show the identical SM cross section contributions. In figure $5(\mathrm{a})$, the top Yukawa coupling is fixed to $y_{t}=y_{t}^{\mathrm{SM}}$, and the bottom Yukawa coupling is varied according to eq. (3.1); in figure $5(\mathrm{~b}), y_{b}=y_{b}^{\mathrm{SM}}$ and $y_{t}$ varies; and in figure $5(\mathrm{c})$, both couplings vary according to eq. (3.1). Obviously, $\sigma_{b \bar{b} Z H}$ (blue/dashdotted) is proportional to $y_{b}^{2}$; even at large $y_{b}$ (small $\kappa$, figure $(\mathrm{a}, \mathrm{c})$ ), it is down by almost a factor of 20 relative to the DY contribution (green/dotted).

The dependence of the $g g Z H$ contribution (red/dashed) on the bottom Yukawa coupling is very small. Its $y_{t}$ dependence is non-trivial, though, due to interference terms. Over a large range of $y_{t}$, the dominant contribution to $g g Z H$ originates from terms 


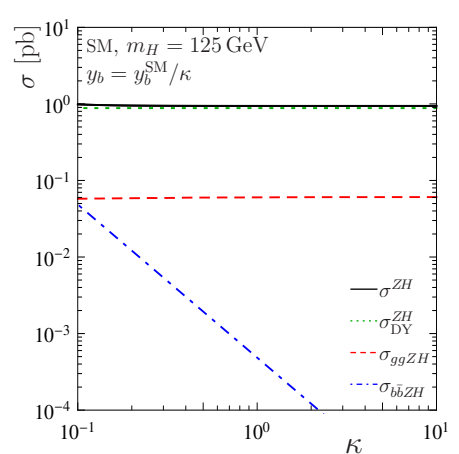

(a)

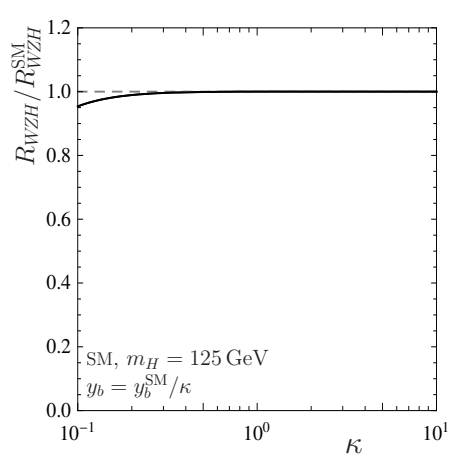

(d)

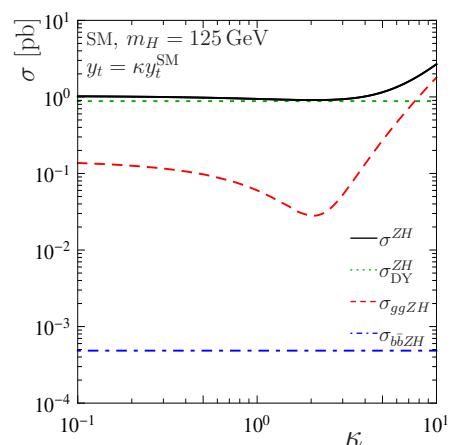

(b)

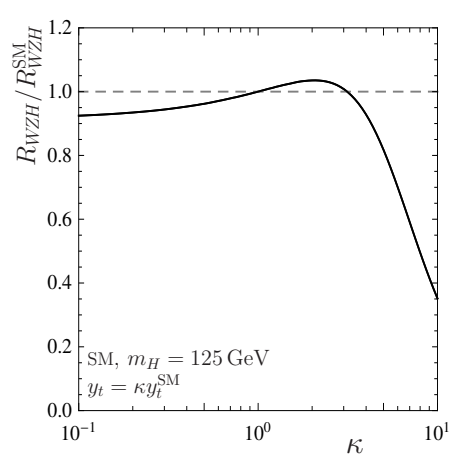

(e)

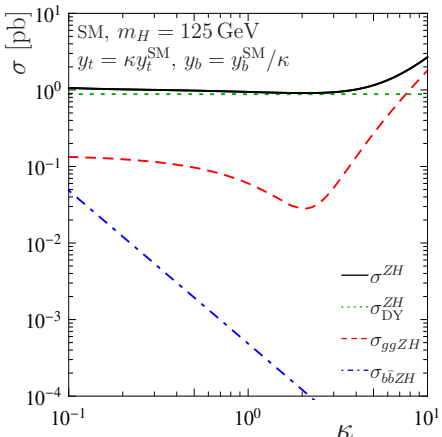

(c)

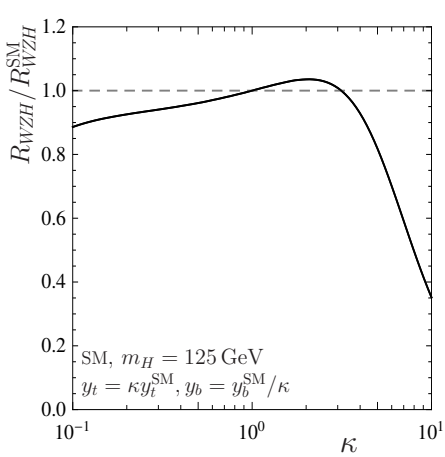

(f)

Figure 5. $(\mathrm{a}-\mathrm{c}) \sigma(p p \rightarrow Z H)$ (black/solid), $\sigma_{\mathrm{DY}}^{Z H}$ (green/dotted), $\sigma_{g g Z H}$ (red/dashed) and $\sigma_{b \bar{b} Z H}$ (blue/dash-dotted) in $\mathrm{pb}$ for $\sqrt{s}=14 \mathrm{TeV}$ and $m_{H}=125 \mathrm{GeV}$ as a function of $\kappa$, where (a) $y_{t}=y_{t}^{\mathrm{SM}}$ and $y_{b}=y_{b}^{\mathrm{SM}} / \kappa$, (b) $y_{t}=\kappa y_{t}^{\mathrm{SM}}$ and $y_{b}=y_{b}^{\mathrm{SM}}$, (c) $y_{t}=\kappa y_{t}^{\mathrm{SM}}$ and $y_{b}=y_{b}^{\mathrm{SM}} / \kappa$; (e-f) the ratio $R_{W Z H} / R_{W Z H}^{\mathrm{SM}}$ for cases (a-c).

$g g \rightarrow Z^{*} \rightarrow Z H$ that do not depend on any Yukawa coupling (see figure $1(\mathrm{~b})$ ). Once $y_{t}$ is larger than about $3 y_{t}^{\mathrm{SM}}$, the $g g Z H$ component rises steeply. It surpasses the DY contribution at about $y_{t}=7 y_{t}^{\mathrm{SM}}$, exceeding it by more than a factor of two at $y_{t}=10 y_{t}^{\mathrm{SM}}$.

The resulting $W H / Z H$ ratios $R_{W Z H}$, normalized to the SM ratio $R_{W Z H}^{\mathrm{SM}}$, are shown as the solid line in figures $5(\mathrm{~d}-\mathrm{f})$. Note that for consistency also the SM result has been obtained according to eq. (2.4) here, with electro-weak corrections and $\mathcal{O}\left(\alpha_{s}^{3}\right)$ effects to $\sigma_{g g Z H}$ neglected. For $\kappa \lesssim 4$, the deviation from the SM prediction (dashed) does not significantly exceed $10 \%$ within the range of $\kappa$ considered here. An increased top Yukawa coupling, however, leads to a sharp decrease of $R_{W Z H}$ : for $\kappa=10$, the value of this ratio is almost three times smaller than its SM prediction.

To conclude this section, let us remark that, apart from the magnitude of the top Yukawa coupling, an anomalous sign of the top Yukawa coupling would be an undoubtful indicator of New Physics. Its measurement clearly requires interference effects; a determination through associated Higgs and single-top production has been suggested in ref. [74, 75].

The $g g \rightarrow Z H$ process offers another, albeit more indirect way to measure the sign of the top Yukawa coupling due to the interference between box- and triangle diagrams (see, 
e.g., figures 1 (b) and (c), respectively) [76]. Since this interference is destructive in the $\mathrm{SM}$, the ratio $R_{W Z H}$ decreases when changing the top Yukawa coupling to its negative. For example, at $m_{H}=125 \mathrm{GeV}$ and $\sqrt{s}=14 \mathrm{TeV}$, the decrease is about $20 \%$ when using the LO result for $g g \rightarrow Z H$; we estimate that this decrease can be as large as $30 \%$ when higher orders are included. In the boosted regime, this effect should be even more prominent due to the enhanced importance of the $g g \rightarrow Z H$ process (see section 5).

While the impact of a sign change in the bottom Yukawa coupling relative to its SM value is rather small, it might be worth investigating this issue as well in extended theories. A more detailed analysis is beyond the scope of this paper though.

\section{2-Higgs-doublet model}

As discussed in section 2.2, the Higgs Strahlung cross section in the 2HDM is not only affected by the modification of the couplings, but also by the contribution from additional Higgs bosons. In this section we will therefore extend the discussion of the previous section to the full $2 \mathrm{HDM}$. We start with a comment on our choice of the 2HDM parameters, followed by a short note about the influence of internal scalars to Higgs Strahlung at the partonic level of the cross section. Then we focus on the production of the light Higgs and subsequently extend our discussion to the heavy and the pseudoscalar Higgs boson. In each case our main concern is the ratio $R_{W Z \phi}$.

As explained before, $\sigma_{\mathrm{I}}^{V \phi}$ and $\sigma_{\mathrm{II}}^{Z \phi}$ are not taken into account in the subsequent discussion of production cross sections in the $2 \mathrm{HDM}$. The contribution $\sigma_{g g Z \phi}$ is calculated at $\mathcal{O}\left(\alpha_{s}^{2}\right), \sigma_{\text {DY }}^{V \phi}$ without electro-weak corrections.

\subsection{Choice of 2HDM parameters}

Apart from the SM input, the Higgs Strahlung cross section depends on the 2HDM Yukawa type (see appendix A) as well as the parameters $\tan \beta, \sin (\beta-\alpha)$, and the masses of the neutral Higgs bosons. If an intermediate Higgs state (see, e.g., figure 3) becomes resonant, the total decay width of that state enters the calculation, see eq. (2.3). This introduces a dependence on the parameter $m_{12}$ which, however, is weak. We therefore set $m_{12}=0$ in all our numerical examples. Note that according to stability, perturbativity, and unitarity requirements, this choice is actually allowed only as long as $\tan \beta \lesssim 1$. Different choices of $m_{12}$ can considerably loosen this constraint though, without affecting our numerical results very much.

Identifying the observed Higgs signal at the LHC with the light Higgs of the 2HDM forces the value of $\sin (\beta-\alpha)$ to be close to 1 . In this paper, we largely disregard such constraints and always scan the full range of $\sin (\beta-\alpha)$.

\section{2 $g g Z \phi$ and $b \bar{b} Z \phi$ contributions involving internal (pseudo)scalars}

As pointed out in section 2.2 , the cross section $p p \rightarrow \phi Z$ in the $2 \mathrm{HDM}$ includes $g g Z \phi$ and $b \bar{b} Z \phi$ contributions that involve an internal scalar different from the final state Higgs particle $\phi$. Before moving on to the full results, let us look at this contribution in a bit more detail. 


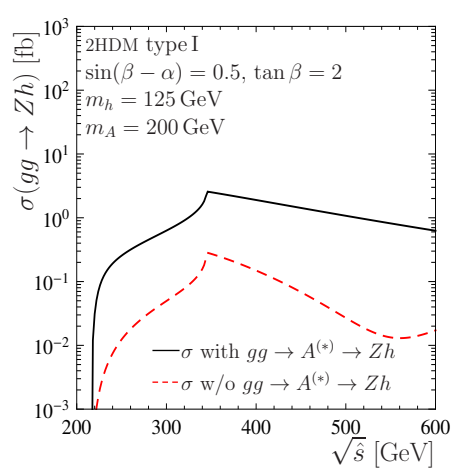

(a)

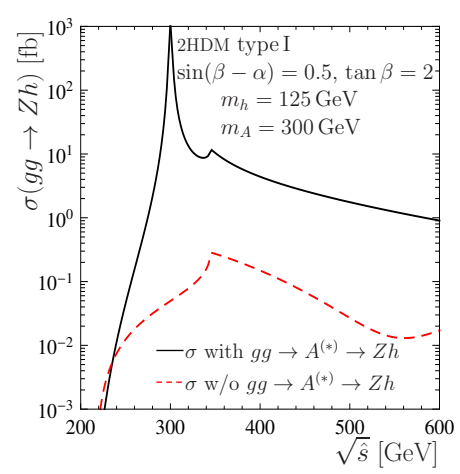

(b)

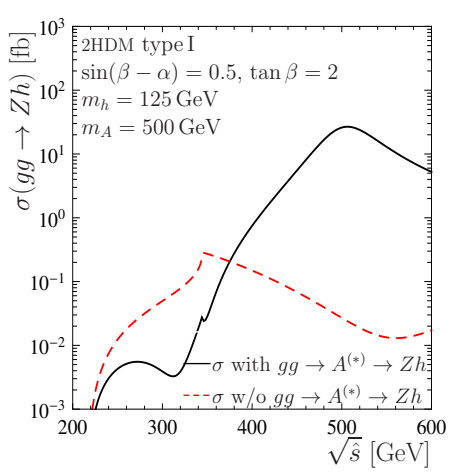

(c)

Figure 6. $\sigma(g g \rightarrow Z h)$ in fb at the partonic level as a function of $\sqrt{\hat{s}}$ in $\mathrm{GeV}$ for $m_{h}=125 \mathrm{GeV}$, $\sin (\beta-\alpha)=0.5$ and $\tan \beta=2$. The red/dashed line corresponds to $\sigma$ without internal pseudoscalar. The black lines show $\sigma$ with the contribution involving an internal pseudoscalar Higgs for (a) $m_{A}=200 \mathrm{GeV}$, (b) $m_{A}=300 \mathrm{GeV}$ and (c) $m_{A}=500 \mathrm{GeV}$.

Consider the production of the light Higgs $h$ with mass $m_{h}=125 \mathrm{GeV}$ for $\sin (\beta-\alpha)=$ 0.5 and $\tan \beta=2$. Figure 6 shows the partonic cross section $g g \rightarrow Z h$ as a function of the partonic cms energy $\sqrt{\hat{s}}$, with (black/solid) and without (red/dashed) the pseudoscalar $A$ as internal particle, for three different values of its mass (a-c). Below the kinematical threshold $\sqrt{\hat{s}}<m_{h}+m_{Z} \approx 216 \mathrm{GeV}$, the $g g Z h$ contribution vanishes. In figure 6 (a), the mass of the pseudoscalar Higgs is $m_{A}=200 \mathrm{GeV}$; it is therefore only produced off-shell. Already then, the numerical contribution to the partonic cross section is quite significant. In figure 6 (b) and (c), on the other hand, the pseudoscalar Higgs is assumed heavier and therefore becomes resonant. For $m_{A}=300 \mathrm{GeV}$ (figure $6(\mathrm{~b})$ ), its total width is $\Gamma_{A}=$ $2.82 \mathrm{GeV}$ which leads to a sharp peak in the partonic cross section. For $m_{A}=500 \mathrm{GeV}$ (figure $6(\mathrm{c})$ ), on the other hand, the corresponding width becomes $\Gamma_{A}=75.0 \mathrm{GeV}$ and the peak is much broader. Independent of $m_{A}$, the heavy and charged Higgs masses are chosen to be $m_{H^{0}}=m_{H^{ \pm}}=200 \mathrm{GeV}$.

All cases of figure 6 show the significance of the internal scalar to the $g g Z$ contribution; a similar discussion applies to $g g Z H^{0}$ and $g g Z A$ production, as well as to the $b \bar{b} Z \phi$ mode. The numerical effects of these terms on the hadronic cross sections and in particular on the ratio $R_{W Z \phi}$ will be discussed in the subsequent section.

\subsection{Light Higgs}

The black solid line in figures 7 (a-c) shows the hadronic cross section $\sigma(p p \rightarrow Z h)$ in pb for $\sqrt{s}=14 \mathrm{TeV}$ and $m_{h}=125 \mathrm{GeV}$ in a type I $2 \mathrm{HDM}$ as a function of $\sin (\beta-\alpha)$ for $\tan \beta=1,5$, and 20 , respectively. The pseudoscalar mass is taken to be $m_{A}=200 \mathrm{GeV}$; the other $2 \mathrm{HDM}$ parameters are irrelevant for this example. The remaining lines in these figures correspond to different contributions entering the total cross section.

The DY terms (green/dotted) are proportional to the square of $g_{V V}^{h}=\sin (\beta-\alpha)$ and do not depend on any other parameters. They are therefore identical in all three cases, vanish at $\sin (\beta-\alpha)=0$, and are symmetric around this point. 


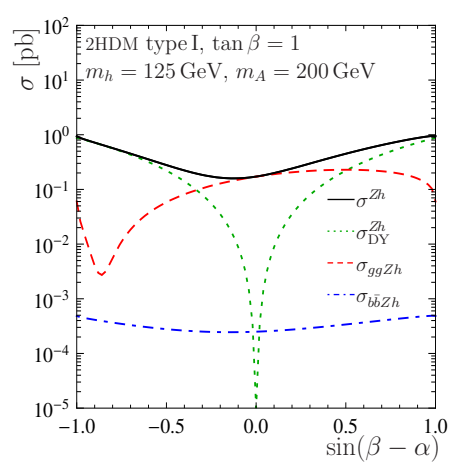

(a)

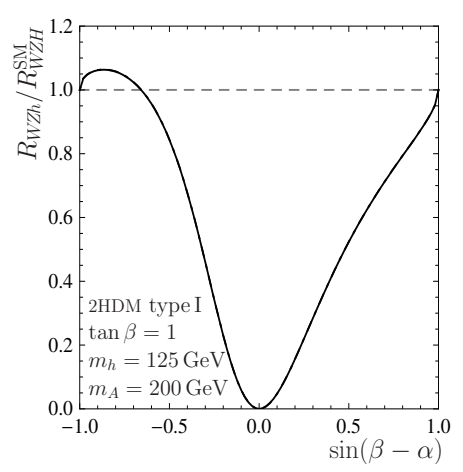

(d)

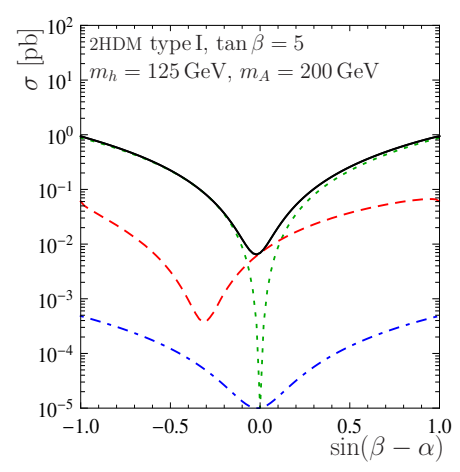

(b)

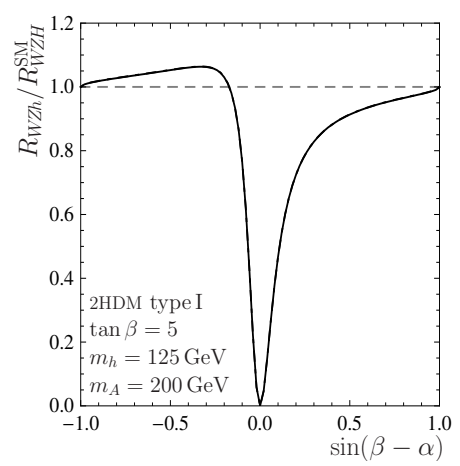

(e)

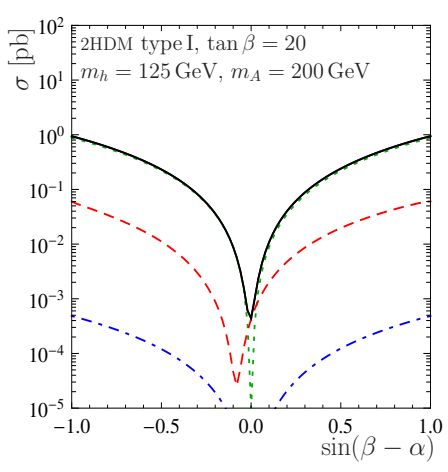

(c)

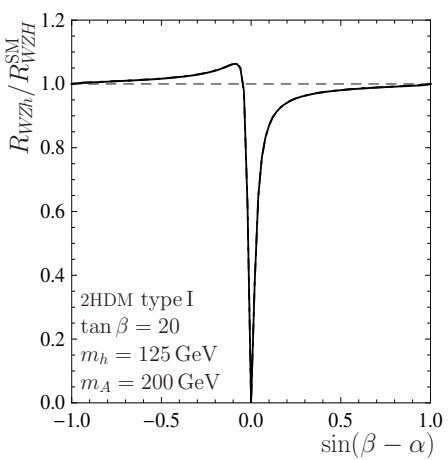

(f)

Figure 7. (a-c) $\sigma(p p \rightarrow Z h)$ (black/solid), $\sigma_{\mathrm{DY}}^{Z h}$ (green/dotted), $\sigma_{g g Z h}$ (red/dashed) and $\sigma_{b \bar{b} Z h}$ (blue/dash-dotted) in pb for $\sqrt{s}=14 \mathrm{TeV}$ and $m_{h}=125 \mathrm{GeV}$ as a function of $\sin (\beta-\alpha)$ for a type I $2 \mathrm{HDM}$ with $m_{A}=m_{H^{0}}=m_{H^{ \pm}}=200 \mathrm{GeV}$ using (a) $\tan \beta=1$, (b) $\tan \beta=5$ and (c) $\tan \beta=20 ;(\mathrm{d}-\mathrm{e})$ the ratio $R_{W Z h} / R_{W Z H}^{\mathrm{SM}}$ for the cases (a-c).

The $g g Z h$ terms are displayed as the red/dashed line; their amplitude involves contributions proportional to $g_{V V}^{h}$ and contributions proportional to the Yukawa couplings $g_{b}^{h}$ and $g_{t}^{h}$. Additionally, the process $g g \rightarrow A^{*} \rightarrow Z h$ is relevant, whose amplitude is proportional to $g_{A Z}^{h}=\cos (\beta-\alpha)$ and $g_{b}^{A}$ or $g_{t}^{A}$. In case of the type I $2 \mathrm{HDM}$, both $g_{b}^{h}$ and $g_{t}^{h}$ (and $g_{b}^{A}$ and $g_{t}^{A}$ ) decrease with increasing $\tan \beta$, which implies that for larger values of $\tan \beta$ the overall cross section $\sigma_{g g Z h}$ is dominated by $g_{V V}^{h}$. For smaller values of $\tan \beta$, the $g g Z h$ terms can become dominant even for rather large values of $|\sin (\beta-\alpha)|$.

The $b \bar{b} Z h$ contribution (blue/dash-dotted) is of no relevance in the type I $2 \mathrm{HDM}$. Note that for $\sin (\beta-\alpha)= \pm 1$, all contributions assume their SM values as then $\cos \alpha / \sin \beta= \pm 1$ and therefore $y_{t, b}= \pm y_{t, b}^{\mathrm{SM}}$ (see appendix A).

Figures 7 (d-f) show the corresponding ratios $R_{W Z h}$ with respect to the SM ratio $R_{W Z H}^{\mathrm{SM}}$ for the three different values of $\tan \beta$ as the solid line by taking into account all available contributions. At $\tan \beta=1$, the ratio exhibits a remarkably strong dependence on $\sin (\beta-$ $\alpha$ ), also close to the edges. Towards larger values of $\tan \beta$, the curves become flatter for $\sin (\beta-\alpha)= \pm 1$ since the $Z h$-specific contributions become more and more suppressed. At $\sin (\beta-\alpha)=0, W h$ production is identical to zero in our approximation (recall that we 


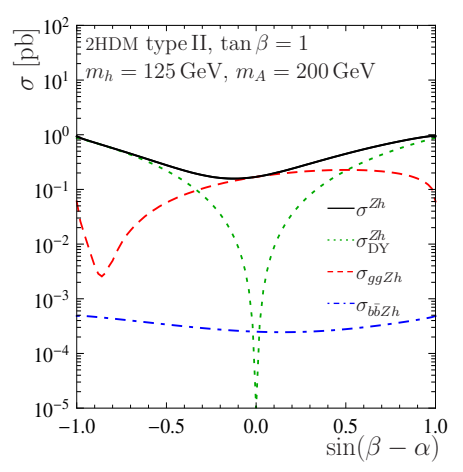

(a)

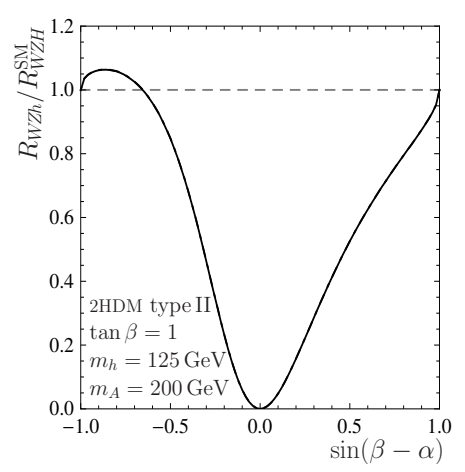

(d)

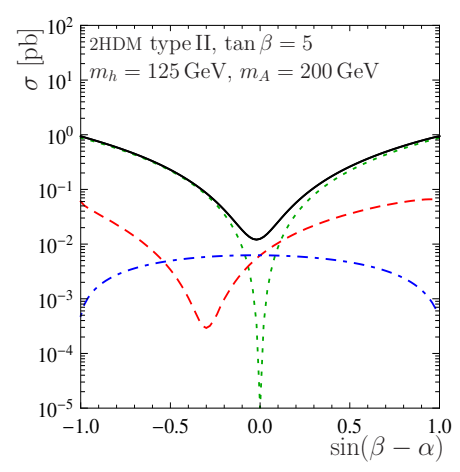

(b)

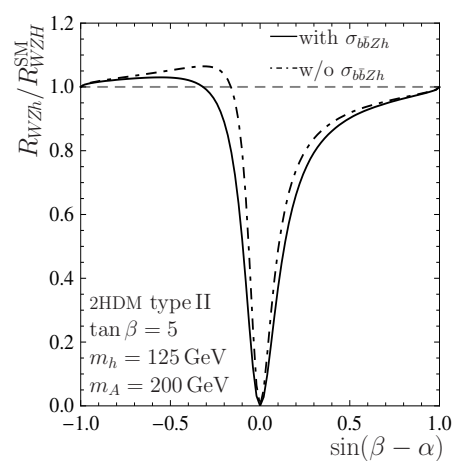

(e)

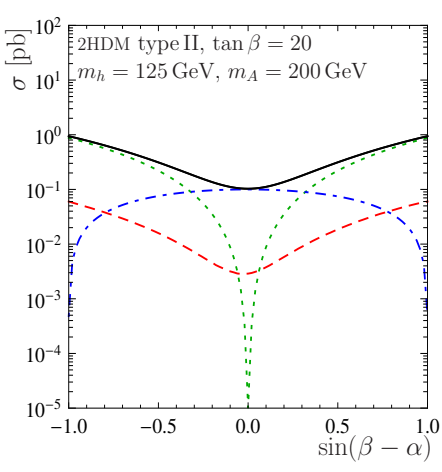

(c)

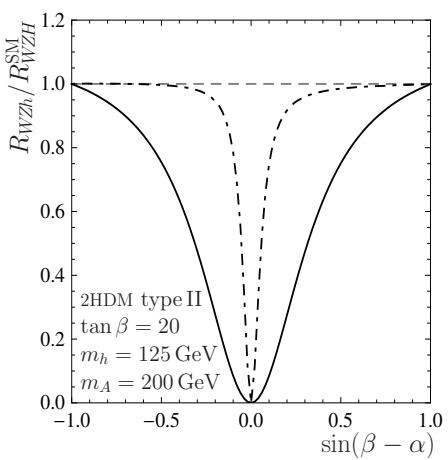

(f)

Figure 8. (a-c) $\sigma(p p \rightarrow Z h)$ (black/solid), $\sigma_{\text {DY }}^{Z h}$ (green/dotted), $\sigma_{g g Z h}$ (red/dashed) and $\sigma_{b \bar{b} Z h}$ (blue/dash-dotted) in pb for $\sqrt{s}=14 \mathrm{TeV}$ and $m_{h}=125 \mathrm{GeV}$ as a function of $\sin (\beta-\alpha)$ for type II $2 \mathrm{HDM}$ with $m_{A}=m_{H^{0}}=m_{H^{ \pm}}=200 \mathrm{GeV}$ using (a) $\tan \beta=1$, (b) $\tan \beta=5$ and (c) $\tan \beta=20 ;(\mathrm{d}-\mathrm{e})$ the ratio $R_{W Z h} / R_{W Z H}^{\mathrm{SM}}$ for the cases (a-c), respectively with $\sigma_{b \bar{b} Z h}$ (solid) and without (dash-dotted).

neglect $\sigma_{\mathrm{I}}^{V h}$ as well as $\sigma_{\mathrm{II}}^{Z h}$ ). Figures 7 (d-f) also include a dash-dotted curve which, for the parameters of this example, is almost indistinguishable from the solid curve. It shows the ratio $R_{W Z h}$ without the $\sigma_{b \bar{b} Z h}$ contribution; this might be a useful quantity if the $b \bar{b} Z \phi$ process can be excluded efficiently by applying a $b$-tagging veto. In the current example, this is irrelevant, of course, but we will use the same notation in other examples, where the $b \bar{b} Z \phi$ process is numerically much more important.

In figure 8 we show the corresponding results for a type II 2HDM. The DY contributions are identical to type I, see figure 7 . For $\tan \beta=1$, also the $g g Z h$ curve is quite similar to the type I case because the $b$-loop contribution is very small in both cases, and the top Yukawa coupling is identical. Since $\sigma_{b \bar{b} Z h}$ is again very small, also the total cross section - and therefore also the ratio $R_{W Z h}$, see figure $8(\mathrm{~d})$ - is almost the same as for type I. In the limit $\sin (\beta-\alpha)= \pm 1$, again all curves assume their SM value for the same reason as discussed above.

With increasing $\tan \beta$, the bottom Yukawa coupling becomes more and more important. For the $g g Z h$ contribution, this results in a complicated interplay with the top Yukawa 


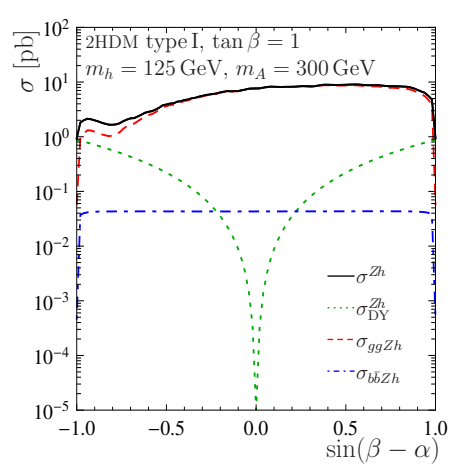

(a)

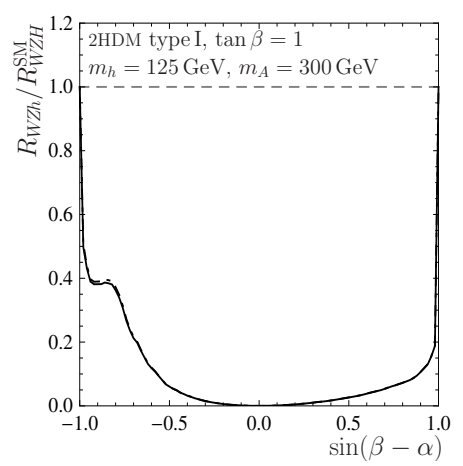

(d)

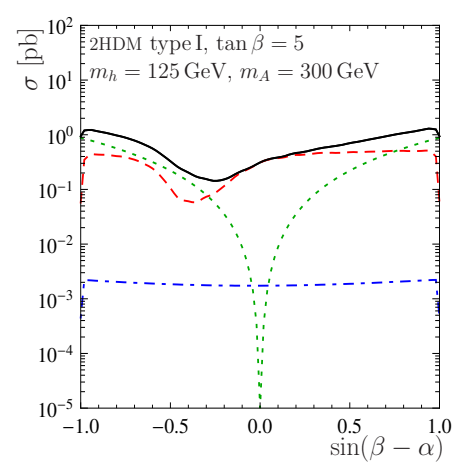

(b)

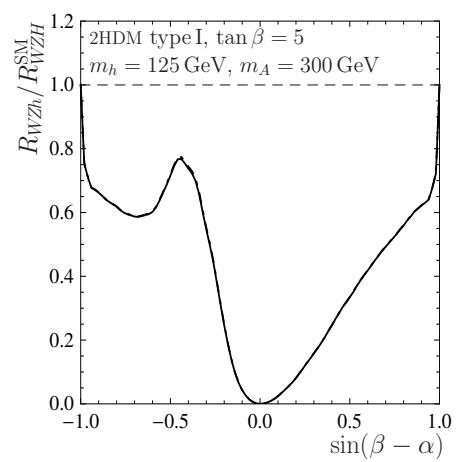

(e)

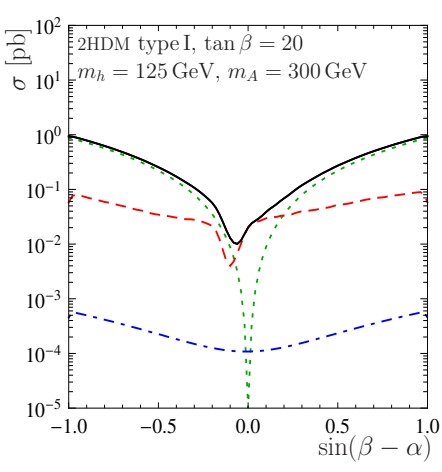

(c)

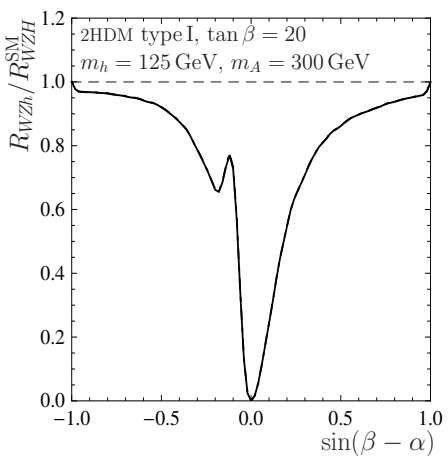

(f)

Figure 9. (a-c) $\sigma(p p \rightarrow Z h)$ (black/solid), $\sigma_{\text {DY }}^{Z h}$ (green/dotted), $\sigma_{g g Z h}$ (red/dashed) and $\sigma_{b \bar{b} Z h}$ (blue/dash-dotted) in pb for $\sqrt{s}=14 \mathrm{TeV}$ and $m_{h}=125 \mathrm{GeV}$ as a function of $\sin (\beta-\alpha)$ for type I $2 \mathrm{HDM}$ with $m_{A}=m_{H^{0}}=m_{H^{ \pm}}=300 \mathrm{GeV}$ using (a) $\tan \beta=1$, (b) $\tan \beta=5$ and (c) $\tan \beta=20$; (d-e) the ratio $R_{W Z h} / R_{W Z H}^{\mathrm{SM}}$ for the cases (a-c).

and the $g g \rightarrow\{Z, A\}^{*} \rightarrow Z h$ terms, which first reduces the $g g Z h$ fraction due to the destructive top-bottom interference, before the bottom effects start to dominate, leading to an increase. The $b \bar{b} Z h$ contribution, on the other hand, increases drastically with $\tan \beta$ and even starts to exceed the other contributions in a wider and wider range of $\sin (\beta-\alpha)$. The importance of this channel is reflected also in the ratio plots figure 8 (d-f): without it (dash-dotted line), the $\sin (\beta-\alpha)$ dependence towards \pm 1 for $\tan \beta>1$ is much flatter than when it is taken into account.

These examples already show that a simple reweighting of the SM cross section by $\left(g_{V V}^{h}\right)^{2}$ (which would resemble the behaviour of the DY contributions in the shown figures) is not appropriate in general. Even in regions where $g_{V V}^{h}$ is close (but not equal) to \pm 1 , the Yukawa contributions to the amplitude proportional to $g_{t}^{h}$ and $g_{b}^{h}$ (and $g_{t}^{A}$ and $g_{b}^{A}$ ) can become non-negligible.

If the mass of the pseudoscalar Higgs exceeds the kinematical threshold, $m_{A}>m_{h}+$ $m_{Z}$, it can be produced on-shell via $g g \rightarrow A \rightarrow Z h$ or $b \bar{b} \rightarrow A \rightarrow Z h$, leading to a substantial increase of these contributions to the cross sections under consideration, see figure 6 . The parameters for figure 9 are identical to those of figure 7 , except that the 
pseudoscalar mass $m_{A}$ is increased from $200 \mathrm{GeV}$ to $300 \mathrm{GeV}$. Since now we have to take into account the total width of the pseudoscalar Higgs, which in turn depends on $m_{12}$ and the heavy and charged Higgs masses $m_{H^{0}}$ and $m_{H^{ \pm}}$, we have to specify these parameters as well. Unless stated otherwise, we will set $m_{12}=0$ and $m_{H^{0}}=m_{H^{ \pm}}=m_{A}$ in what follows.

The DY contributions are obviously not affected by the change in $m_{A}$. For small $\tan \beta$ and $\sin (\beta-\alpha) \neq \pm 1$, the resonant $g g \rightarrow A \rightarrow Z h$ process dramatically increases the $g g Z h$ contribution, making it dominant by more than a factor of 10 in most of the $\sin (\beta-\alpha)$ region. The fact that it features a relatively constant behaviour for most values of $\alpha$ can be understood as follows: for small values of $\tan \beta$, the amplitude is approximately proportional to $\left(g_{Z}^{A h}\right)^{2}=\cos ^{2}(\beta-\alpha)$. Squaring the propagator of eq. (2.3) results in a Breit-Wigner function. If we write the latter function in the narrow-width approximation

$$
\frac{1}{\left(\hat{s}-m_{A}^{2}\right)^{2}+m_{A}^{2} \Gamma_{A}^{2}} \rightarrow \frac{\pi}{m_{A} \Gamma_{A}} \delta\left(\hat{s}-m_{A}^{2}\right),
$$

the factor $\cos ^{2}(\beta-\alpha)$ is canceled by $1 / \Gamma_{A}$ as long as the decay mode $A \rightarrow Z h$ is dominant. For $|\sin (\beta-\alpha)| \rightarrow 1$ the decay mode $A \rightarrow Z h$ is suppressed. As soon as its partial width drops below the other partial widths, mainly $\Gamma(A \rightarrow b \bar{b})$, the contribution $\sigma_{g g Z h}$ is instantly turning towards its SM value. In case $m_{A}>2 m_{t}$ the decay channel $A \rightarrow t \bar{t}$ opens and lowers the pseudoscalar contributions. For increasing $\tan \beta$, the suppression of $g_{t}^{A}$ and $g_{b}^{A}$ reduces the impact of the pseudoscalar as internal particle. A similar statement holds for the contribution $\sigma_{b \bar{b} Z \mathbf{Z}}$, but their contribution is down by a factor $10^{-2}$ to $10^{-3}$.

Similarly to the case $m_{A}=200 \mathrm{GeV}$, the impact of the $g g Z h$ and $b \bar{b} Z h$ terms decreases towards larger $\tan \beta$ and the total cross section is described by the pure DY terms better and better. For $\tan \beta \lesssim 5$, however the ratio $R_{W Z h}$ significantly deviates from its SM value even in the regions very close to $\sin (\beta-\alpha)= \pm 1$ (see figures $9(\mathrm{~d}, \mathrm{e})$ ). In fact, for $\tan \beta=1$, $R_{W Z h}<0.7$ (meaning $R_{W Z h} / R_{W Z h}^{\mathrm{SM}}<0.4$ ) for $-0.91<\sin (\beta-\alpha)<0.99$.

Figure 10 shows the cross section dependence on $\sin (\beta-\alpha)$ for a type II 2HDM. The parameters are the same as in figure 8 , except that the masses $m_{A}=m_{H^{0}}=m_{H^{ \pm}}$are increased from $200 \mathrm{GeV}$ to $300 \mathrm{GeV}$. Similarly to the case for $m_{A}=200 \mathrm{GeV}$, the curve for $\tan \beta=1$ very much resembles the one for type I, which is again due to the little impact of the bottom contributions, of course. Towards larger values of $\tan \beta$, the $b \bar{b} Z h$ fraction becomes dominant very quickly. The behaviour of the ratio $R_{W Z h}$ is thus quite remarkable for all values of $\tan \beta$, see figures 10 (d-f). If the $b \bar{b} Z h$ component is disregarded (for reasons discussed above), the deviation of $R_{W Z h}$ from its SM value becomes less drastic towards larger $\tan \beta$, in particular close to $\sin (\beta-\alpha)= \pm 1$, but it still amounts to about $20 \%$ for $|\sin (\beta-\alpha)|<0.9$ at $\tan \beta=5$.

The statements of this section are also valid in case of other choices of the parameters, in particular for other choices of $m_{12}$, since $m_{12}$ only affects the decay widths of the internal pseudoscalar, as discussed above. If however the heavy Higgs and/or charged Higgs bosons are light enough to open the decay channels $A \rightarrow Z H^{0}$ and/or $A \rightarrow W^{ \pm} H^{\mp}$ in addition, the decay width of $A$ is enlarged and thus the $g g Z h$ and $b \bar{b} Z h$ cross section contributions get reduced. We will discuss this effect in the subsequent section for the heavy Higgs, where $A \rightarrow Z h, A \rightarrow Z H^{0}$ and $A \rightarrow W^{ \pm} H^{\mp}$ are possible. 


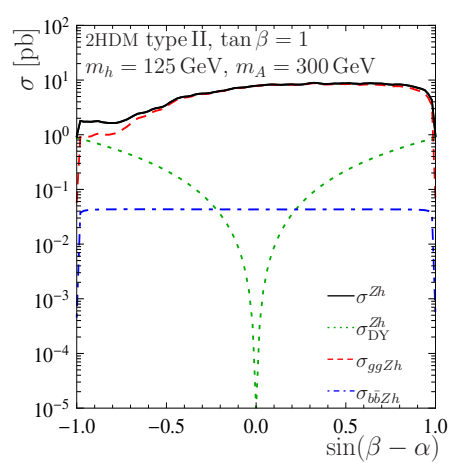

(a)

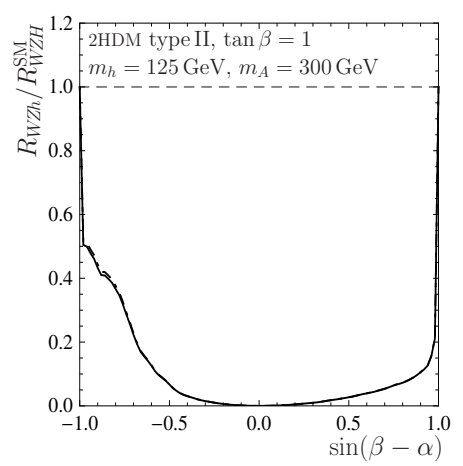

(d)

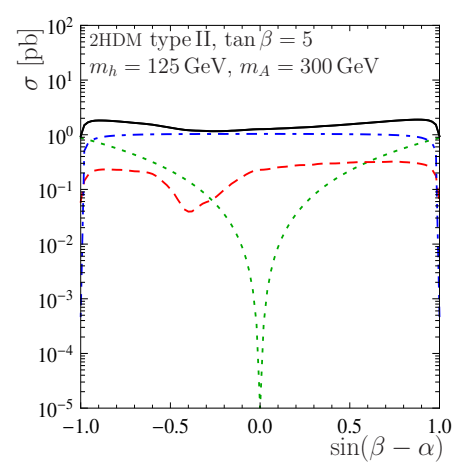

(b)

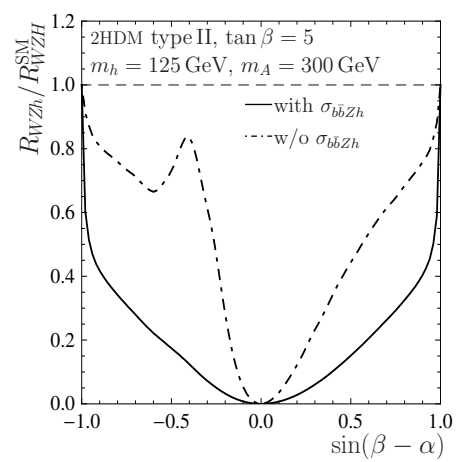

(e)

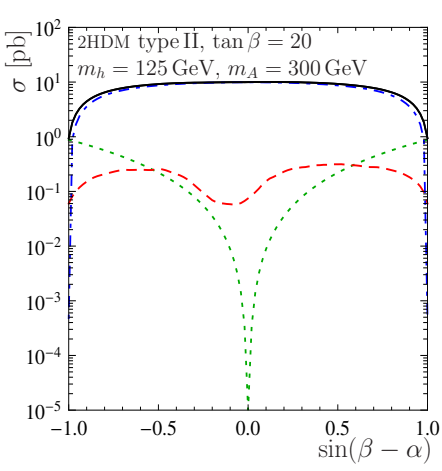

(c)

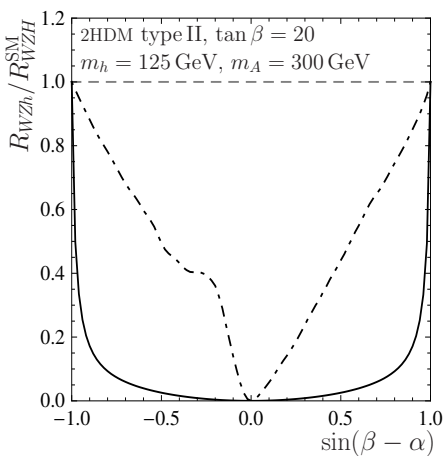

(f)

Figure 10. (a-c) $\sigma(p p \rightarrow Z h)$ (black/solid), $\sigma_{\mathrm{DY}}^{Z h}$ (green/dotted), $\sigma_{g g Z h}$ (red/dashed) and $\sigma_{b \bar{b} Z h}$ (blue/dash-dotted) in pb for $\sqrt{s}=14 \mathrm{TeV}$ and $m_{h}=125 \mathrm{GeV}$ as a function of $\sin (\beta-\alpha)$ for type II $2 \mathrm{HDM}$ with $m_{A}=m_{H^{0}}=m_{H^{ \pm}}=300 \mathrm{GeV}$ using (a) $\tan \beta=1$, (b) $\tan \beta=5$ and (c) $\tan \beta=20$; (d-e) the ratio $R_{W Z h} / R_{W Z H}^{\mathrm{SM}}$ for the cases $(\mathrm{a}-\mathrm{c})$.

\subsection{Heavy and pseudoscalar Higgs}

The heavy Higgs couples to the heavy gauge bosons proportional to $g_{V V}^{H^{0}}=\cos (\beta-\alpha)$. Therefore, if the light Higgs resembles the SM-like Higgs, the Drell-Yan-like contributions $\sigma_{\mathrm{DY}}^{V H^{0}}$ are strongly suppressed. In contrast, the Yukawa couplings are still sizable and therefore dominate this production process in the region $|\sin (\beta-\alpha)| \approx 1$. In figure 11 , we show the different contributions to the cross section for $p p \rightarrow Z H^{0}$ in the type II 2HDM when the mass of the pseudoscalar Higgs is below the $Z H^{0}$ threshold $\left(m_{H^{0}}=m_{A}=200 \mathrm{GeV}\right)$. figure 12 , on the other hand, demonstrates the presence of a resonance $\left(m_{H^{0}}=200 \mathrm{GeV}\right.$, $\left.m_{A}=300 \mathrm{GeV}\right)$. In contrast to the cases discussed in section 4.3 , the internal pseudoscalar can then decay via $A \rightarrow Z h, A \rightarrow Z H^{0}$ and $A \rightarrow W^{ \pm} H^{\mp}$. Thus, the $g g Z H^{0}$ and $b \bar{b} Z H^{0}$ contributions are not constant, but the proportionality to $\left(g_{Z}^{A} H^{0}\right)^{2}=\sin ^{2}(\beta-\alpha)$ dominates.

Figure $11(\mathrm{c}-\mathrm{d})$ and figure $12(\mathrm{c}-\mathrm{d})$ present the ratios $R_{W Z H^{0}}$ with respect to the SM ratio. For $|\sin (\beta-\alpha)| \approx 1$ the cross section $\sigma^{W H^{0}}$ vanishes, whereas $\sigma^{Z H^{0}}$ is dominated by Yukawa couplings, such that $R_{W Z H^{0}}$ tends towards zero. The detailed measurement of $\sigma^{Z H^{0}}$ therefore provides important information about the Yukawa couplings of the heavy Higgs in case the light Higgs resembles the SM-like Higgs. The latter effect is less important in case of type I $2 \mathrm{HDM}$ with large values of $\tan \beta$, since all Yukawa couplings are suppressed. 


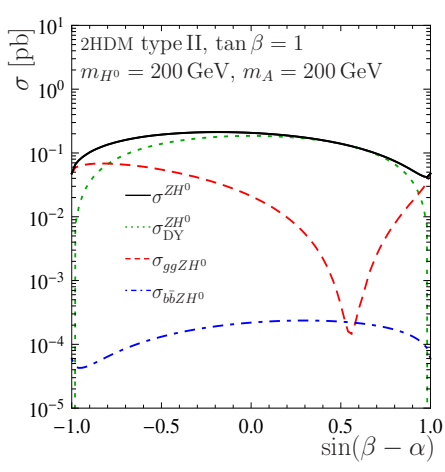

(a)

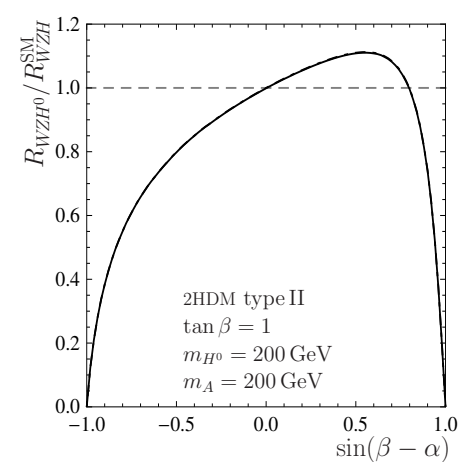

(c)

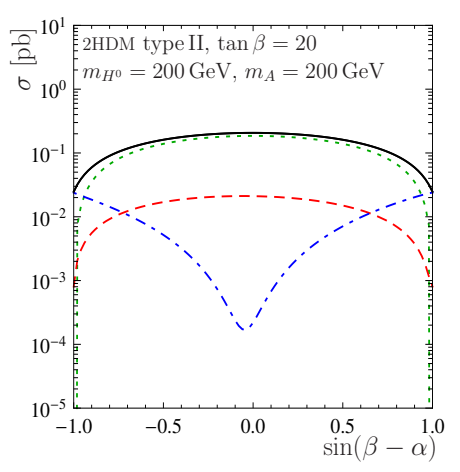

(b)

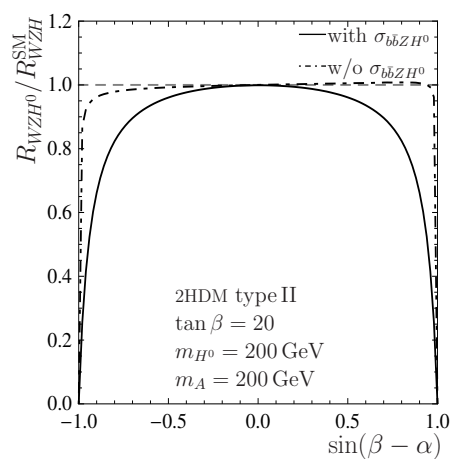

(d)

Figure 11. (a-b) $\sigma\left(p p \rightarrow Z H^{0}\right)$ (black/solid), $\sigma_{\text {DY }}^{Z H^{0}}$ (green/dotted), $\sigma_{g g Z H^{0}}$ (red/dashed) and $\sigma_{b \bar{b} Z H^{0}}$ (blue/dash-dotted) in pb for $\sqrt{s}=14 \mathrm{TeV}$ and $m_{H^{0}}=200 \mathrm{GeV}$ as a function of $\sin (\beta-\alpha)$ for type II $2 \mathrm{HDM}$ with $m_{h}=125 \mathrm{GeV}, m_{A}=m_{H^{ \pm}}=200 \mathrm{GeV}$ using (a) $\tan \beta=1$ and (b) $\tan \beta=20 ;(\mathrm{c}-\mathrm{d})$ the ratio $R_{W Z H^{0}} / R_{W Z H}^{\mathrm{SM}}$ for the cases $(\mathrm{a}-\mathrm{b})$, respectively with $\sigma_{b \bar{b} Z H^{0}}$ (solid) and without (dash-dotted).

The pseudoscalar Higgs of the 2HDM does not couple to the heavy gauge bosons, since $g_{V V}^{A}=0$. However, the presence of Yukawa couplings $g_{t}^{A}$ and $g_{b}^{A}$ allows for large $g g Z A$ and $b \bar{b} Z A$ cross section contributions. Figure 13 (a) demonstrates the size of both $g g Z A$ and $b \bar{b} Z A$ cross section contributions to $Z A$ production in the type II $2 \mathrm{HDM}$ with $\tan \beta=20$. As expected, the cross sections are hardly dependent on the mixing angle $\alpha$. However, figure 13 (b) shows the presence of a heavy scalar $H^{0}$, which can decay to $Z A$. In this case, the cross sections are slightly increased and a dependence on the mixing angle $\alpha$ is induced. Since we omit $\sigma_{\mathrm{I}}^{W A}$, the production cross section $\sigma(p p \rightarrow W A)$ vanishes and a definition of $R_{W Z A}$ is not reasonable for the pseudoscalar.

\section{Effects in the boosted regime}

\subsection{The gluon-induced component in the SM}

It is well known that the signal-to-background ratio for $V H$ production is significantly enhanced in the so-called boosted regime [52], where the transverse momentum $p_{T}^{H}$ of the 


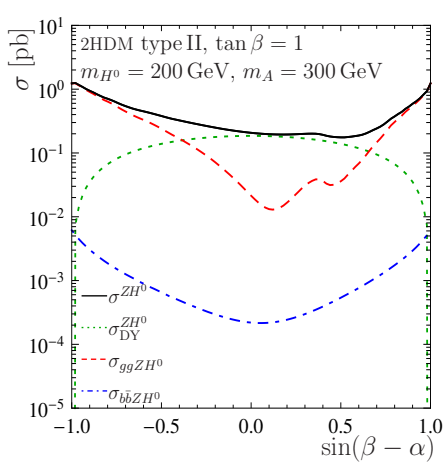

(a)

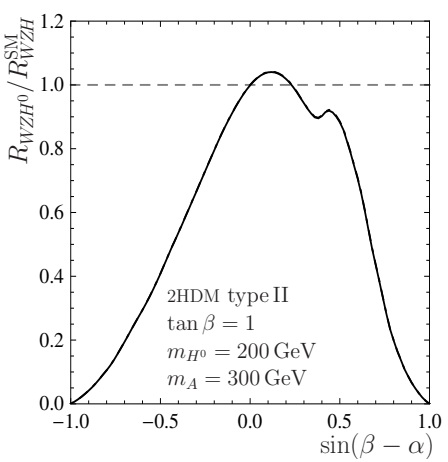

(c)

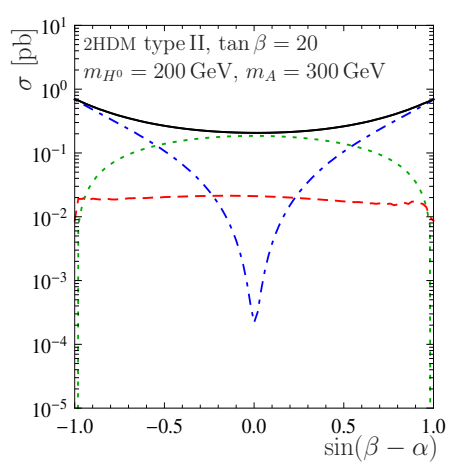

(b)

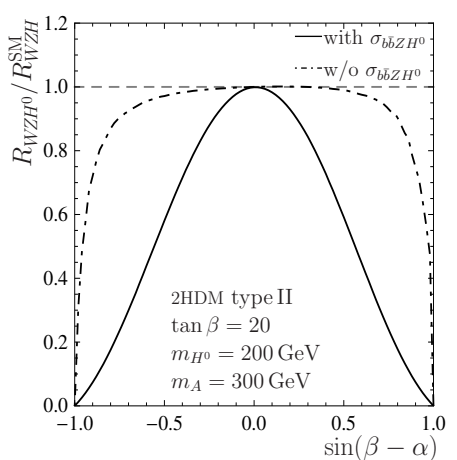

(d)

Figure 12. (a-b) $\sigma\left(p p \rightarrow Z H^{0}\right)$ (black/solid), $\sigma_{\mathrm{DY}}^{Z H^{0}}$ (green/dotted), $\sigma_{g g Z H^{0}}$ (red/dashed) and $\sigma_{b \bar{b} Z H^{0}}$ (blue/dash-dotted) in pb for $\sqrt{s}=14 \mathrm{TeV}$ and $m_{H^{0}}=200 \mathrm{GeV}$ as a function of $\sin (\beta-\alpha)$ for type II $2 \mathrm{HDM}$ with $m_{h}=125 \mathrm{GeV}, m_{A}=300 \mathrm{GeV}, m_{H^{ \pm}}=200 \mathrm{GeV}$ using (a) $\tan \beta=1$ and (b) $\tan \beta=20$; (c-d) the ratio $R_{W Z H^{0}} / R_{W Z H}^{\mathrm{SM}}$ for the cases (a-b), respectively with $\sigma_{b \bar{b} Z H^{0}}$ (solid) and without (dash-dotted).

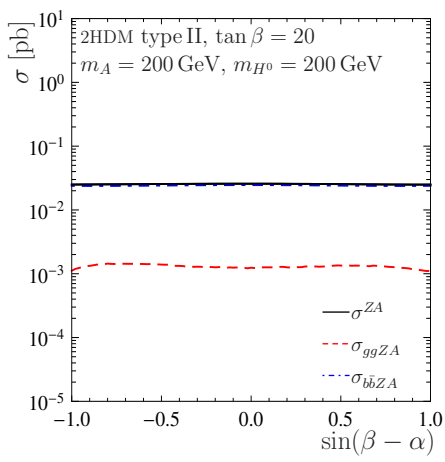

(a)

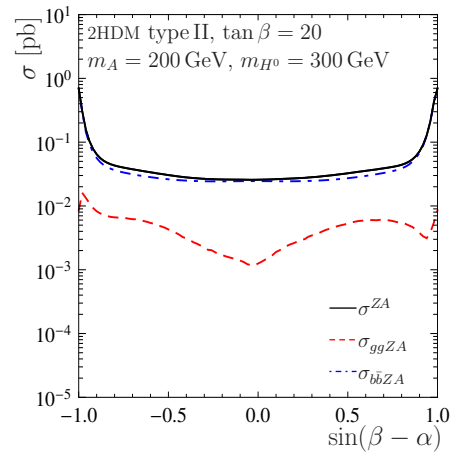

(b)

Figure 13. (a-b) $\sigma(p p \rightarrow Z A)$ (black/solid), $\sigma_{g g Z A}$ (red/dashed) and $\sigma_{b \bar{b} Z A}$ (blue/dash-dotted) in pb for $\sqrt{s}=14 \mathrm{TeV}$ and $m_{A}=200 \mathrm{GeV}$ as a function of $\sin (\beta-\alpha)$ for type II 2HDM with $\tan \beta=20, m_{h}=125 \mathrm{GeV}, m_{H^{ \pm}}=200 \mathrm{GeV}$ using (a) $m_{H^{0}}=200 \mathrm{GeV}$ and (b) $m_{H^{0}}=300 \mathrm{GeV}$. 


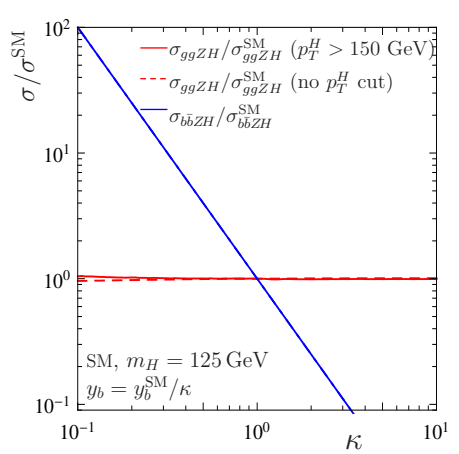

(a)

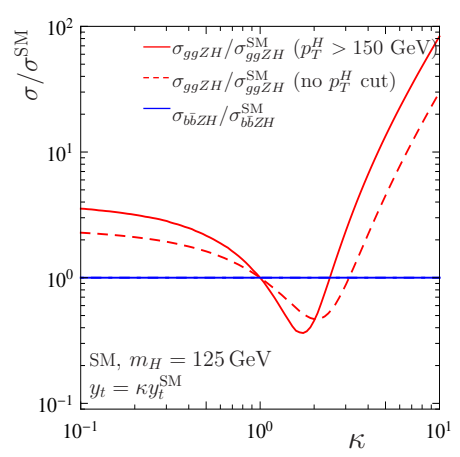

(b)

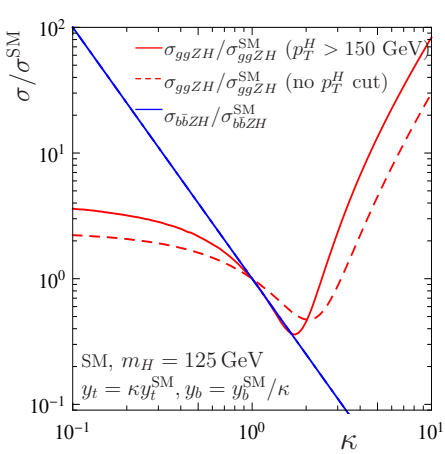

(c)

Figure 14. (a-c) $\sigma_{g g Z H} / \sigma_{g g Z H}^{\mathrm{SM}}(\mathrm{red}), \sigma_{b \bar{b} Z H} / \sigma_{b \bar{b} Z H}^{\mathrm{SM}}$ (blue) with $p_{T}^{H}>150 \mathrm{GeV}$ (solid) and without $p_{T}^{H}$ cut (dashed) for $\sqrt{s}=14 \mathrm{TeV}$ and $m_{H}=125 \mathrm{GeV}$ as a function of $\kappa$, where (a) $y_{t}=y_{t}^{\mathrm{SM}}$ and $y_{b}=y_{b}^{\mathrm{SM}} / \kappa$, (b) $y_{t}=\kappa y_{t}^{\mathrm{SM}}$ and $y_{b}=y_{b}^{\mathrm{SM}}$, (c) $y_{t}=\kappa y_{t}^{\mathrm{SM}}$ and $y_{b}=y_{b}^{\mathrm{SM}} / \kappa$.

Higgs boson is large. In this section, we therefore briefly study the influence of a lower cut on $p_{T}^{H}$ on the effects observed in the previous sections. The discussion in this section will be on a rather qualitative level; more quantitative studies are beyond the scope of our paper and will be deferred to a future publication.

First we note that in the SM, the Higgs transverse momentum due to the $g g \rightarrow Z H$ sub-process is peaked at larger values than when the Higgs is produced through the DY-like process (see, e.g., ref. [76]). The effect of this is that the relative $g g \rightarrow Z H$ contribution is about twice as large as for the total cross section if one restricts the Higgs transverse momentum to $p_{T}^{H} \gtrsim 150 \mathrm{GeV}$ [77] (assuming $m_{H}=125 \mathrm{GeV}$ ). Increasing the lower cut on $p_{T}^{H}$ beyond that value leads again to a decrease of the relative $g g \rightarrow Z H$ portion since the spectrum drops rather sharply towards large $p_{T}^{H}$.

The second step is to study the effect of New Physics on the $g g \rightarrow Z \phi$ and the $b \bar{b} \rightarrow Z \phi$ sub-processes in the boosted regime. The shape of the $p_{T}^{\phi}$ spectrum is clearly unaffected for the DY-like terms in the scenarios considered in sections 3 and 4 of this paper.

\subsection{Modified SM Yukawa couplings}

Figure 14 shows the $g g \rightarrow Z H$ (red) and the $b \bar{b} \rightarrow Z H$ contribution (blue) relative to the SM one for modified top- and bottom-Yukawa couplings (parameters as in figure 5) without a $p_{T}^{H}$ cut (dashed), and when the Higgs transverse momentum is restricted to $p_{T}^{H}>150 \mathrm{GeV}$ (solid). Since $\sigma_{b \bar{b} Z H}$ is proportional to $\left|y_{b}\right|^{2}$ in the SM, the corresponding dashed and solid line are identical.

For $g g \rightarrow Z H$, on the other hand, it is remarkable that almost any modification of the top-Yukawa coupling leads to an increase in the large- $p_{T}^{H}$ fraction with respect to the SM one (solid vs. dashed). A decrease is only observed for $y_{t}^{\mathrm{SM}}<y_{t}<2 y_{t}^{\mathrm{SM}}$. Additionally, close to the SM value $y_{t} \approx y_{t}^{\mathrm{SM}}$, the dependence on $\kappa$ and thus on the top-Yukawa coupling is increased. Combined with the observations of a generally enhanced SM $g g \rightarrow Z H$ contribution with respect to the DY-terms, we conclude that the $W H / Z H$ ratio becomes significantly more sensitive to the top-Yukawa coupling in the boosted regime. 


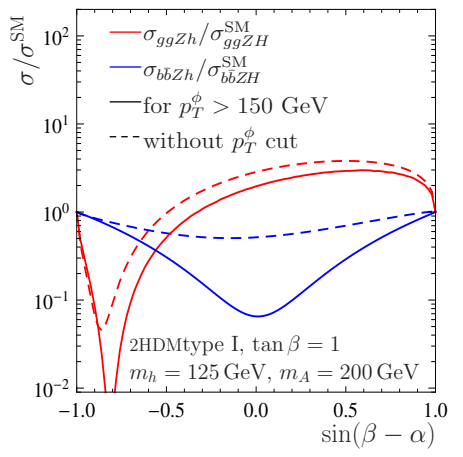

(a)

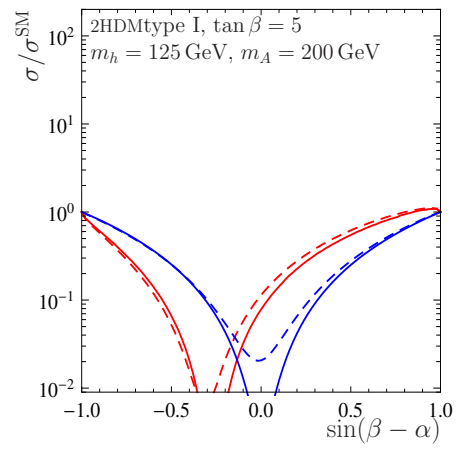

(b)

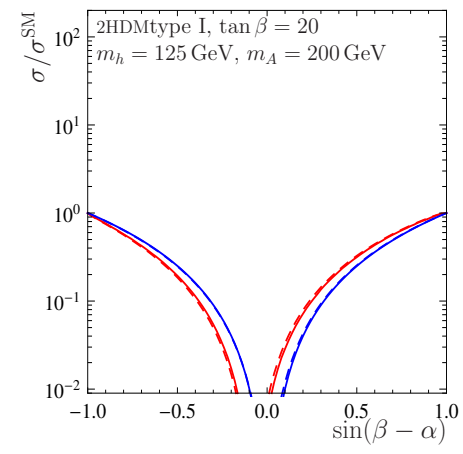

(c)

Figure 15. (a-c) $\sigma_{g g Z h} / \sigma_{g g Z H}^{\mathrm{SM}}(\mathrm{red}), \sigma_{b \bar{b} Z h} / \sigma_{b \bar{b} Z H}^{\mathrm{SM}}$ (blue) with $p_{T}^{\phi}>150 \mathrm{GeV}$ (solid) and without $p_{T}^{\phi}$ cut (dashed) for $\sqrt{s}=14 \mathrm{TeV}$ in the $2 \mathrm{HDM}$ type I with $m_{A}=200 \mathrm{GeV}$ as a function of $\sin (\beta-\alpha)$ using (a) $\tan \beta=1$, (b) $\tan \beta=5$ and (c) $\tan \beta=20$.

\subsection{2-Higgs-doublet model}

Let us now turn from the simple modification of the Yukawa couplings to the full 2HDM. The main new effects here are due to the additional Higgs bosons $\phi^{\prime}$ which can occur as virtual particles. Their impact is particularly large when values $\hat{s} \approx m_{\phi^{\prime}}^{2}$ are kinematically allowed, see section 2.2. Applying a lower cut on $p_{T}^{\phi}$ restricts the values of $\hat{s}$ to

$$
\hat{s} \geq m_{\phi}^{2}+m_{Z}^{2}+2\left(p_{T}^{\phi}\right)^{2}+2 \sqrt{\left(m_{Z}^{2}+\left(p_{T}^{\phi}\right)^{2}\right)\left(m_{\phi}^{2}+\left(p_{T}^{\phi}\right)^{2}\right)} .
$$

For example, while the lower limit on $\sqrt{\hat{s}}$ for the inclusive cross section is at $m_{Z}+m_{\phi} \approx$ $216 \mathrm{GeV}$ for $m_{\phi}=125 \mathrm{GeV}$, it moves up to about $370 \mathrm{GeV}$ in the boosted regime with $p_{T}^{\phi}>150 \mathrm{GeV}$. We therefore expect that the effects on the $W \phi / Z \phi$ ratio observed in section 4 decrease in the boosted regime.

First we consider the case corresponding to figure 7, i.e. $2 \mathrm{HDM}$ type I with $m_{A}=$ $200 \mathrm{GeV}$. Note that for the total inclusive cross section, the pseudoscalar is already slightly below resonant; a lower cut on $p_{T}^{\phi}$ moves the allowed interval for $\hat{s}$ further away from the resonance. Figure 15 shows the contributions of the subprocesses $g g \rightarrow Z h$ (red) and $b \bar{b} \rightarrow Z h$ (blue) in the 2HDM relative to the SM quantities in this case. For the solid lines, a lower cut on $p_{T}^{\phi}$ ( $\phi=h$ for the $2 \mathrm{HDM}, \phi=H$ for the SM) of $150 \mathrm{GeV}$ was applied, while this cut is absent for the dashed lines. The effect of the cut is non-negligible only for the case $\tan \beta=1$, where it leads to a moderate decrease of the $g g \rightarrow Z h$ fraction relative to the SM. The $b \bar{b} \rightarrow Z h$ is completely negligible in this scenario anyway (see figure 7 ), so the reduction by the $p_{T}^{\phi}$ cut observed in figure 15 is irrelevant.

For the $g g \rightarrow Z h$ component, the situation looks similar also in the 2HDM type II with non-resonant pseudoscalar (see figure 16; parameters as in figure 8). For moderate to large $\tan \beta$, the effect of the $p_{T}^{\phi}$-cut on the $b \bar{b} \rightarrow Z h$ component, on the other hand, is quite drastic, leading to a reduction of about an order of magnitude.

The largest impact of a restriction to large $p_{T}^{\phi}$, however, is expected in scenarios where this cut removes effects from resonant intermediate particles. In fact, this is what can be 


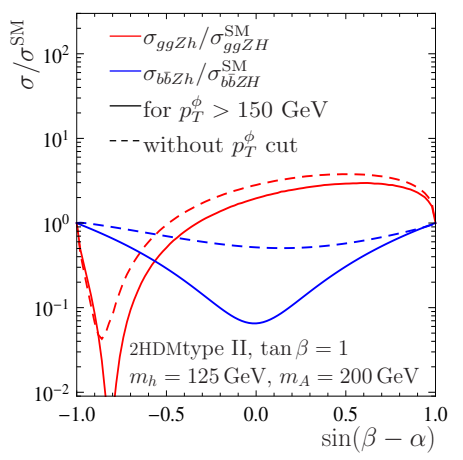

(a)

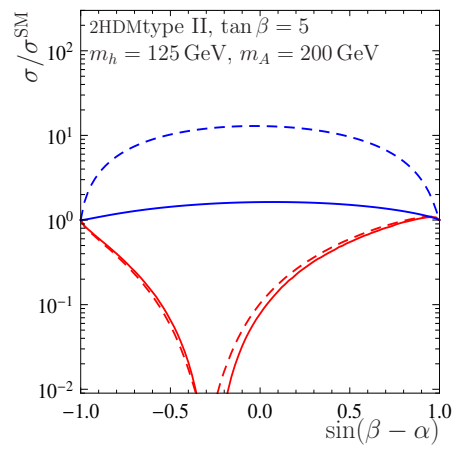

(b)

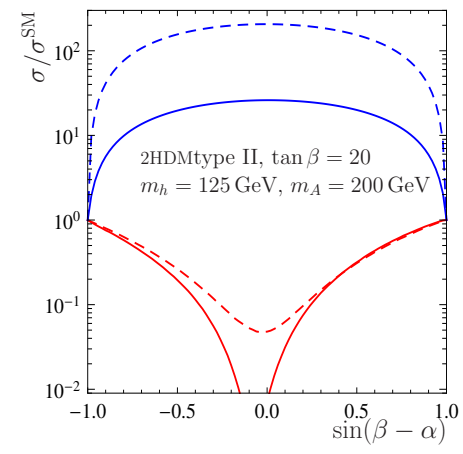

(c)

Figure 16. (a-c) $\sigma_{g g Z h} / \sigma_{g g Z H}^{\mathrm{SM}}(\mathrm{red}), \sigma_{b \bar{b} Z h} / \sigma_{b \bar{b} Z H}^{\mathrm{SM}}$ (blue) with $p_{T}^{\phi}>150 \mathrm{GeV}$ (solid) and without $p_{T}^{\phi}$ cut (dashed) for $\sqrt{s}=14 \mathrm{TeV}$ in the $2 \mathrm{HDM}$ type II with $m_{A}=200 \mathrm{GeV}$ as a function of $\sin (\beta-\alpha)$ using (a) $\tan \beta=1$, (b) $\tan \beta=5$ and (c) $\tan \beta=20$.

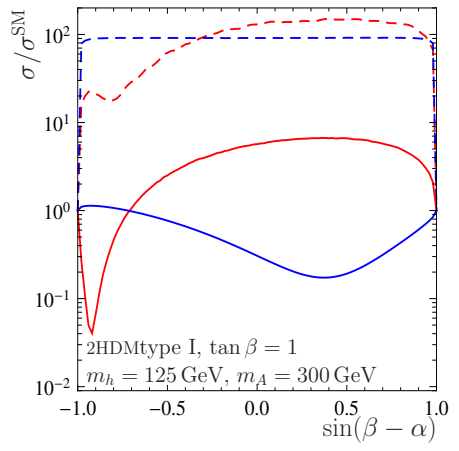

(a)

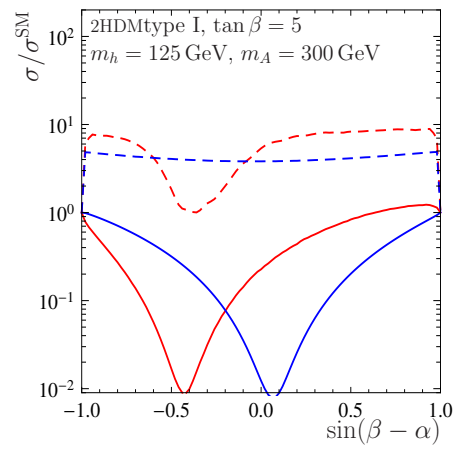

(b)

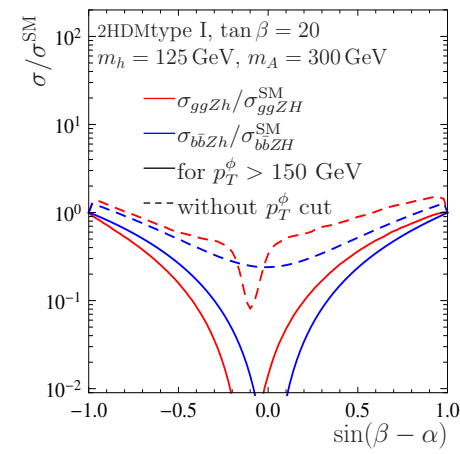

(c)

Figure 17. (a-c) $\sigma_{g g Z h} / \sigma_{g g Z H}^{\mathrm{SM}}(\mathrm{red}), \sigma_{b \bar{b} Z h} / \sigma_{b \bar{b} Z H}^{\mathrm{SM}}$ (blue) with $p_{T}^{\phi}>150 \mathrm{GeV}$ (solid) and without $p_{T}^{\phi}$ cut (dashed) for $\sqrt{s}=14 \mathrm{TeV}$ in the $2 \mathrm{HDM}$ type I with $m_{A}=300 \mathrm{GeV}$ as a function of $\sin (\beta-\alpha)$ using (a) $\tan \beta=1$, (b) $\tan \beta=5$ and (c) $\tan \beta=20$.

observed in figures 17 and 18, which correspond to the parameter sets of figures 9 and 10, respectively, i.e. $2 \mathrm{HDM}$ type I and II with pseudoscalar mass $m_{A}=300 \mathrm{GeV}$. Without $p_{T}^{\phi}$ cut, the pseudoscalar can become resonant, as discussed in section 4 . If the events are restricted to $p_{T}^{\phi}>150 \mathrm{GeV}$, this is no longer the case. The $g g \rightarrow Z h$ and $b \bar{b} \rightarrow Z h$ fractions are thus diminished, in some cases by more than two orders of magnitude. The missing resonant contribution also explains why the respective solid curves for $m_{A}=200 \mathrm{GeV}$ and $m_{A}=300 \mathrm{GeV}$ in figures 15 and 17 as well as in figures 16 and 18 resemble each other.

Quite generally, we conclude from this discussion that a restriction to the boosted regime may not be advantageous when searching for effects of New Physics. A deterioration of the signal-to-background ratio in the inclusive cross section may well be compensated by effects that are otherwise cut away. 


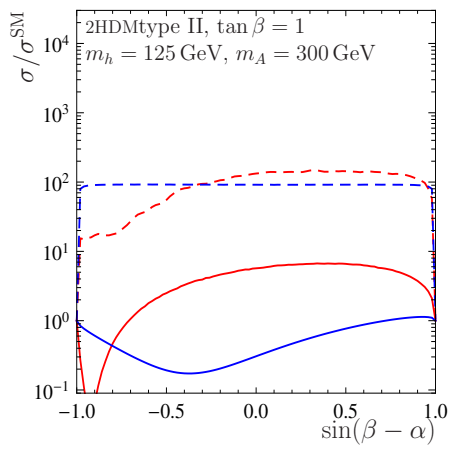

(a)

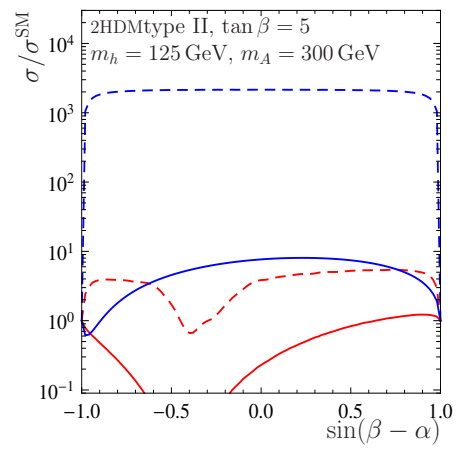

(b)

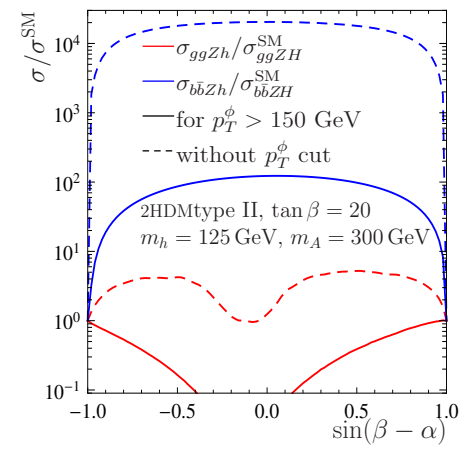

(c)

Figure 18. (a-c) $\sigma_{g g Z h} / \sigma_{g g Z H}^{\mathrm{SM}}\left(\right.$ red), $\sigma_{b \bar{b} Z h} / \sigma_{b \bar{b} Z H}^{\mathrm{SM}}$ (blue) with $p_{T}^{\phi}>150 \mathrm{GeV}$ (solid) and without $p_{T}^{\phi}$ cut (dashed) for $\sqrt{s}=14 \mathrm{TeV}$ in the $2 \mathrm{HDM}$ type II with $m_{A}=300 \mathrm{GeV}$ as a function of $\sin (\beta-\alpha)$ using (a) $\tan \beta=1$, (b) $\tan \beta=5$ and (c) $\tan \beta=20$.

\section{Conclusions}

We provided the SM prediction for the ratio of the cross sections for Higgs production in association with a $W$ and with a $Z$ boson, including all available and numerically relevant theoretical contributions: the Drell-Yan-like terms including NNLO QCD and NLO electroweak corrections, the so-called top-loop induced terms, as well as gluon-initiated effects through NLO. The residual theoretical uncertainty on this ratio is about $3 \%$, induced by missing higher orders, variations in the PDFs, and the experimental error on $\alpha_{s}\left(m_{Z}\right)$.

The gluon-induced terms only affect $Z H$ production, and since they are rather sensitive to New Physics effects, the $W H / Z H$ ratio provides a useful test of the Standard Model, once statistics of the collected data is sufficiently large. As an example, we have considered a general 2HDM where it turns out that intermediate Higgs bosons can give a particularly large contribution to $Z \phi$ production. In addition, bottom quark annihilation becomes numerically relevant and in some cases even dominant. The consequence is a significant drop of the $W \phi / Z \phi$ ratio $R_{W Z \phi}$ as compared to its SM value, even close to the value $\sin (\beta-\alpha)=1$ which seems to be preferred by the recent LHC results. This is even more so when the intermediate Higgs can become resonant. Restriction to the "boosted Higgs"-regime carries the potential danger of cutting away such resonant contributions; we therefore suggest to apply dedicated analyses also on the inclusive cross sections.

There is a number of ways to extend our study, which we leave for future investigations. Let us name just a few:

- In this paper, we focussed on total inclusive $W \phi$ and $Z \phi$ production, with only a rather qualitative consideration of the boosted-Higgs regime. Quite generally, restriction to particular kinematical regions or distributions may further improve the experimental and possibly also the theoretical significance of the effects observed here.

- In a more detailed phenomenological analysis, the decay of the final state Higgs boson $\phi$ has to be folded in. This does not affect the quantity $R_{W Z \phi}$ itself, of course, but 
its experimental sensitivity. Note, however, that for $m_{h}=125 \mathrm{GeV}$, the branching ratio to $b \bar{b}$ is significant unless the bottom Yukawa coupling becomes exceptionally small. Therefore, this main decay mode should be accessible in most of the 2HDM parameter space.

- Clearly, the $W \phi / Z \phi$ ratio should be studied also in other extensions of the SM, for example in supersymmetric models. Due to the tight restrictions on the parameters of the Higgs sector, we expect the effects in the MSSM to be substantially reduced compared to our findings for the 2HDM though.

- It was found that the $\mathcal{O}\left(\alpha_{s}^{3}\right)$ effects to the gluon fusion contribution $\sigma_{g g Z H}$ in the SM are quite substantial [55]. A precise prediction of the $W \phi / Z \phi$ ratio in other models therefore requires the analogous corrections in these models. Similarly, the terms $\sigma_{\mathrm{I}}^{V \phi}$ and $\sigma_{\mathrm{II}}^{Z \phi}$ could receive non-negligible contributions from bottom-quark loops if the bottom Yukawa coupling is large. The relevant calculations are non-trivial, however.

Finally, let us point out that a new release of the program vh@nnlo will include all of the 2HDM effects discussed in this paper and will allow for a flexible calculation of the Higgs Strahlung cross section in the 2HDM. Similarly, for the gluon fusion process $g g \rightarrow \phi$ and bottom quark annihilation $b \bar{b} \rightarrow \phi$, a link of SusHi [78] to 2HDMC will soon be available. Since $t \bar{t} \phi$ production is typically suppressed in $2 \mathrm{HDM}$ models, and a rather good approximation for weak boson fusion is obtained by a simple rescaling of the SM cross section by $g_{V V}^{\phi}$, all relevant cross section for Higgs production at the LHC within a 2HDM will then be available.

Note added. While this paper was in the reviewing process which led us to include section 5, ref. [76] appeared which touches upon similar issues.

\section{Acknowledgments}

We would like to thank the organizers and the participants of the informal meetings during the last few months on 2HDM Higgs production which has inspired this work. In particular, this includes S. Bolognesi, H. Haber, H. Logan, M. Mühlleitner, K. Peters, N. Rompotis, O. Stål, and R. Tanaka. In addition, we would like to thank O. Stål for providing us with a version of 2HDMC which can be linked to SusHi and vh@nnlo.

This work was supported by DFG, contract HA 2990/5-1.

\section{A 2-Higgs-doublet model}

The 2-Higgs-doublet model (2HDM), which contains two Higgs doublets named $H_{1}$ and $H_{2}$, can be cast in four types, if CP conservation and no tree-level flavor-changing neutral currents are demanded. They differ by the Higgs-fermion Yukawa couplings. By convention, $H_{2}$ couples to the up-type quarks, whereas the couplings to the down-type quarks as well as to the leptons depend on the type of $2 \mathrm{HDM}$ and can be taken from table 2. Our notation follows refs. [20-25]. 


\begin{tabular}{|c|c|c|c|c|}
\hline Model & Type I & Type II & Lepton-specific & Flipped \\
\hline up-type quarks & $H_{2}$ & $H_{2}$ & $H_{2}$ & $H_{2}$ \\
down-type quarks & $H_{2}$ & $H_{1}$ & $H_{2}$ & $H_{1}$ \\
leptons & $H_{2}$ & $H_{1}$ & $H_{1}$ & $H_{2}$ \\
\hline
\end{tabular}

Table 2. 2HDM types. In the main text of this paper, we only show results for "type I" and "type II"; the results for "Lepton-specific" and "Flipped" are identical to these, respectively.

Note that type I and Lepton-specific as well as type II and Flipped 2HDM only differ in their lepton couplings. The calculation of our paper does not involve these couplings, though, which is why it is sufficient to restrict ourselves to type I and type II.

Due to CP conservation, the two Higgs doublets form two CP-even Higgs fields $h$ and $H^{0}$ and one CP-odd field $A$. In the "physical basis", their masses, the ratio of the vacuum expectation values $\tan \beta=v_{2} / v_{1}$, and the CP-even Higgs mixing angle $\alpha$ are considered as independent input parameters. In addition, two charged Higgs fields $H^{ \pm}$are formed. The two angles $\alpha$ and $\beta$ determine the relative strength of all couplings with respect to the SM couplings. We follow ref. [20] and present the Feynman rules required in our calculation:
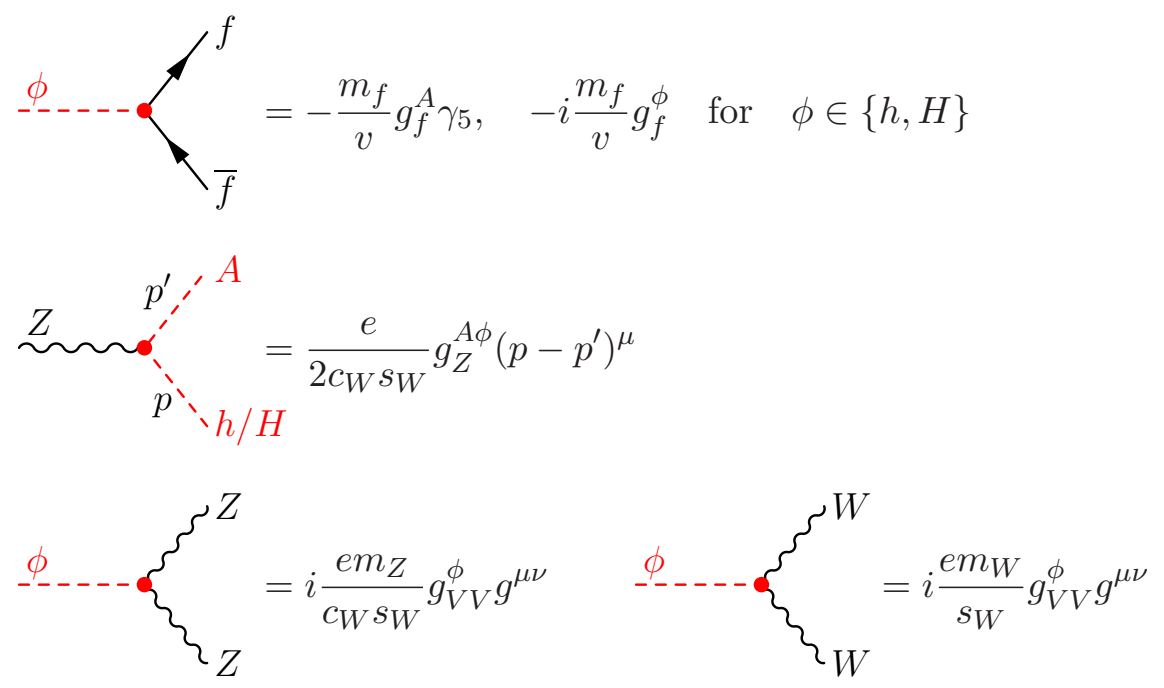

All momenta are considered as incoming, $s_{W}$ and $c_{W}$ denote the sine and cosine of the weak mixing angle, $e$ the electromagnetic charge, and $v=\sqrt{v_{1}^{2}+v_{2}^{2}}=1 / \sqrt{\sqrt{2} G_{F}}$ the SM vacuum expectation value. The relative couplings $g_{f}^{\phi}$ which enter the Yukawa couplings to the quarks, differ in the four 2HDM types and can be taken from table 3 .

In contrast, the relative strength of the couplings to the heavy gauge bosons are independent of the 2HDM type. They are given by

$$
g_{V V}^{h}=\sin (\beta-\alpha), \quad g_{V V}^{H^{0}}=\cos (\beta-\alpha), \quad g_{V V}^{A}=0,
$$

where $V$ represents one of the heavy gauge bosons $V \in\{W, Z\}$, and by

$$
g_{Z}^{A h}=\cos (\beta-\alpha), \quad g_{Z}^{A H^{0}}=-\sin (\beta-\alpha) .
$$




\begin{tabular}{|c|c|c|c|c|}
\hline Model & Type I & Type II & Lepton-specific & Flipped \\
\hline$g_{u}^{h}$ & $\cos \alpha / \sin \beta$ & $\cos \alpha / \sin \beta$ & $\cos \alpha / \sin \beta$ & $\cos \alpha / \sin \beta$ \\
$g_{d}^{h}$ & $\cos \alpha / \sin \beta$ & $-\sin \alpha / \cos \beta$ & $\cos \alpha / \sin \beta$ & $-\sin \alpha / \cos \beta$ \\
$g_{u}^{H}$ & $\sin \alpha / \sin \beta$ & $\sin \alpha / \sin \beta$ & $\sin \alpha / \sin \beta$ & $\sin \alpha / \sin \beta$ \\
$g_{d}^{H}$ & $\sin \alpha / \sin \beta$ & $\cos \alpha / \cos \beta$ & $\sin \alpha / \sin \beta$ & $\cos \alpha / \cos \beta$ \\
$g_{u}^{A}$ & $\cot \beta$ & $\cot \beta$ & $\cot \beta$ & $\cot \beta$ \\
$g_{d}^{A}$ & $-\cot \beta$ & $\tan \beta$ & $-\cot \beta$ & $\tan \beta$ \\
\hline
\end{tabular}

Table 3. Relative couplings $g_{f}^{\phi}$ with respect to the SM Yukawa coupling for the four 2HDM types.

Obviously, the coupling of the light Higgs $h$ to the gauge bosons equals the SM coupling for $\sin (\beta-\alpha)=1$, where also the coupling to the pseudoscalar Higgs vanishes. For convenience of the reader, we add the relative Yukawa couplings in terms of $\sin (\beta-\alpha)$ :

$$
\begin{array}{ll}
\frac{\cos \alpha}{\sin \beta}=\sin (\beta-\alpha)+\cos (\beta-\alpha) \frac{1}{\tan \beta}, & \frac{\sin \alpha}{\sin \beta}=-\sin (\beta-\alpha) \frac{1}{\tan \beta}+\cos (\beta-\alpha) \\
\frac{\cos \alpha}{\cos \beta}=\sin (\beta-\alpha) \tan \beta+\cos (\beta-\alpha), & \frac{\sin \alpha}{\cos \beta}=-\sin (\beta-\alpha)+\cos (\beta-\alpha) \tan \beta .
\end{array}
$$

Open Access. This article is distributed under the terms of the Creative Commons Attribution License (CC-BY 4.0), which permits any use, distribution and reproduction in any medium, provided the original author(s) and source are credited.

\section{References}

[1] ATLAS collaboration, Observation of a new particle in the search for the standard model Higgs boson with the ATLAS detector at the LHC, Phys. Lett. B 716 (2012) 1 [arXiv: 1207.7214] [INSPIRE].

[2] CMS collaboration, Observation of a new boson at a mass of $125 \mathrm{GeV}$ with the CMS experiment at the LHC, Phys. Lett. B 716 (2012) 30 [arXiv:1207.7235] [InSPIRE].

[3] LHC Higgs Cross Section Working Group collaboration, S. Heinemeyer et al., Handbook of LHC Higgs cross sections: 3. Higgs properties, arXiv:1307.1347 [INSPIRE].

[4] Tevnph (Tevatron New Phenomina and Higgs Working Group), CDF, D0 collaboration, Combined CDF and DO search for standard model Higgs boson production with up to $10.0 \mathrm{fb}^{-1}$ of data, arXiv:1203.3774 [INSPIRE].

[5] ATLAS collaboration, Search for associated production of the Higgs boson in the

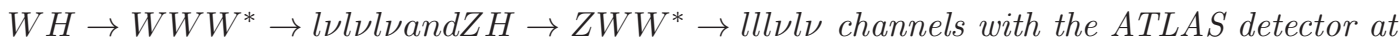
the LHC, ATLAS-CONF-2013-075 (2013).

[6] ATLAS collaboration, Search for the bb decay of the standard model Higgs boson in associated W/ZH production with the ATLAS detector, ATLAS-CONF-2013-079 (2013).

[7] ATLAS collaboration, Search for invisible decays of a Higgs boson produced in association with a $Z$ boson in ATLAS, ATLAS-CONF-2013-011 (2013). 
[8] CMS collaboration, Search for the standard model Higgs boson produced in association with $W$ or $Z$ bosons, and decaying to bottom quarks for LHCp 2013, CMS-PAS-HIG-13-012 (2013).

[9] CMS collaboration, Search for invisible Higgs produced in association with a $Z$ boson, CMS-PAS-HIG-13-018 (2013).

[10] CMS collaboration, VH with $H \rightarrow W W \rightarrow \ell \nu \ell \nu$ and $V \rightarrow j j$, CMS-PAS-HIG-13-017 (2013).

[11] T. Han and S. Willenbrock, $Q C D$ correction to the $p p \rightarrow W H$ and $Z H$ total cross-sections, Phys. Lett. B 273 (1991) 167 [inSPIRE].

[12] O. Brein, A. Djouadi and R. Harlander, NNLO QCD corrections to the Higgs-strahlung processes at hadron colliders, Phys. Lett. B 579 (2004) 149 [hep-ph/0307206] [inSPIRE].

[13] M. Ciccolini, S. Dittmaier and M. Krämer, Electroweak radiative corrections to associated WH and ZH production at hadron colliders, Phys. Rev. D 68 (2003) 073003 [hep-ph/0306234] [INSPIRE].

[14] S. Dawson, T. Han, W. Lai, A. Leibovich and I. Lewis, Resummation effects in vector-boson and Higgs associated production, Phys. Rev. D 86 (2012) 074007 [arXiv:1207.4207] [INSPIRE].

[15] G. Ferrera, M. Grazzini and F. Tramontano, Associated WH production at hadron colliders: a fully exclusive QCD calculation at NNLO, Phys. Rev. Lett. 107 (2011) 152003 [arXiv: 1107.1164] [INSPIRE].

[16] A. Denner, S. Dittmaier, S. Kallweit and A. Muck, Electroweak corrections to Higgs-strahlung off $W / Z$ bosons at the Tevatron and the LHC with HAWK, JHEP 03 (2012) 075 [arXiv:1112.5142] [INSPIRE].

[17] S. Dittmaier et al., Handbook of LHC Higgs cross sections: 2. Differential distributions, arXiv: 1201.3084 [INSPIRE].

[18] D.A. Dicus and C. Kao, Higgs boson- $Z^{0}$ production from gluon fusion, Phys. Rev. D 38 (1988) 1008 [Erratum ibid. D 42 (1990) 2412] [INSPIRE].

[19] B.A. Kniehl, Associated production of Higgs and $Z$ bosons from gluon fusion in hadron collisions, Phys. Rev. D 42 (1990) 2253 [INSPIRE].

[20] J.F. Gunion, H.E. Haber, G.L. Kane and S. Dawson, The Higgs hunter's guide, Front. Phys. 80 (2000) 1 [INSPIRE].

[21] A. Akeroyd, Nonminimal neutral Higgs bosons at LEP-2, Phys. Lett. B 377 (1996) 95 [hep-ph/9603445] [INSPIRE].

[22] A. Akeroyd, Fermiophobic and other nonminimal neutral Higgs bosons at the LHC, J. Phys. G 24 (1998) 1983 [hep-ph/9803324] [INSPIRE].

[23] M. Aoki, S. Kanemura, K. Tsumura and K. Yagyu, Models of Yukawa interaction in the two Higgs doublet model and their collider phenomenology, Phys. Rev. D 80 (2009) 015017 [arXiv:0902.4665] [INSPIRE].

[24] G. Branco et al., Theory and phenomenology of two-Higgs-doublet models, Phys. Rept. 516 (2012) 1 [arXiv:1106.0034] [INSPIRE].

[25] N. Craig and S. Thomas, Exclusive signals of an extended Higgs sector, JHEP 11 (2012) 083 [arXiv: 1207.4835] [INSPIRE]. 
[26] C.-Y. Chen, S. Dawson and M. Sher, Heavy Higgs searches and constraints on two Higgs doublet models, Phys. Rev. D 88 (2013) 015018 [arXiv: 1305.1624] [INSPIRE].

[27] D.S. Alves, P.J. Fox and N.J. Weiner, Higgs signals in a type I 2HDM or with a sister Higgs, arXiv: 1207.5499 [INSPIRE].

[28] N. Craig et al., Multi-lepton signals of multiple Higgs bosons, JHEP 02 (2013) 033 [arXiv: 1210.0559] [INSPIRE].

[29] Y. Bai, V. Barger, L.L. Everett and G. Shaughnessy, The 2HDM-X and Large Hadron Collider data, Phys. Rev. D 87 (2013) 115013 [arXiv:1210.4922] [inSPIRE].

[30] A. Azatov and J. Galloway, Electroweak symmetry breaking and the Higgs boson: confronting theories at colliders, Int. J. Mod. Phys. A 28 (2013) 1330004 [arXiv:1212.1380] [InSPIRE].

[31] P. Ferreira, R. Santos, H.E. Haber and J.P. Silva, Mass-degenerate Higgs bosons at $125 \mathrm{GeV}$ in the two-Higgs-doublet model, Phys. Rev. D 87 (2013) 055009 [arXiv:1211.3131] [INSPIRE].

[32] A. Celis, V. Ilisie and A. Pich, LHC constraints on two-Higgs doublet models, JHEP 07 (2013) 053 [arXiv:1302.4022] [INSPIRE].

[33] B. Grinstein and P. Uttayarat, Carving out parameter space in type-II two Higgs doublets model, JHEP 06 (2013) 094 [Erratum ibid. 1309 (2013) 110] [arXiv:1304.0028] [inSPIRE].

[34] M. Krawczyk, D. Sokołowska and B. Swiezewska, 2HDM with $Z_{2}$ symmetry in light of new LHC data, J. Phys. Conf. Ser. 447 (2013) 012050 [arXiv: 1303.7102] [InSPIRE].

[35] W. Altmannshofer, S. Gori and G.D. Kribs, A minimal flavor violating 2HDM at the LHC, Phys. Rev. D 86 (2012) 115009 [arXiv: 1210.2465] [INSPIRE].

[36] C.-W. Chiang and K. Yagyu, Implications of Higgs boson search data on the two-Higgs doublet models with a softly broken $Z_{2}$ symmetry, JHEP 07 (2013) 160 [arXiv:1303.0168] [INSPIRE].

[37] A. Barroso, P. Ferreira, R. Santos, M. Sher and J.P. Silva, 2HDM at the LHC - The story so far, arXiv: 1304.5225 [INSPIRE].

[38] N. Craig, J. Galloway and S. Thomas, Searching for signs of the second Higgs doublet, arXiv:1305.2424 [INSPIRE].

[39] C.-Y. Chen and S. Dawson, Exploring two Higgs doublet models through Higgs production, Phys. Rev. D 87 (2013) 055016 [arXiv:1301.0309] [inSPIRE].

[40] O. Eberhardt, U. Nierste and M. Wiebusch, Status of the two-Higgs-doublet model of type-II, JHEP 07 (2013) 118 [arXiv: 1305.1649] [INSPIRE].

[41] P. Ferreira, R. Santos, M. Sher and J.P. Silva, 2HDM confronting LHC data, arXiv: 1305.4587 [INSPIRE].

[42] ATLAS collaboration, Search for Higgs bosons in two-Higgs-doublet models in the $H \rightarrow W W \rightarrow e \nu \mu \nu$ channel with the ATLAS detector, ATLAS-CONF-2013-027 (2013).

[43] A. Djouadi, The anatomy of electro-weak symmetry breaking. II. The Higgs bosons in the minimal supersymmetric model, Phys. Rept. 459 (2008) 1 [hep-ph/0503173] [INSPIRE].

[44] C. Kao, Production of a pseudoscalar Higgs with a $Z$ boson from gluon fusion, Phys. Rev. D 46 (1992) 4907 [inSPIRE]. 
[45] J. Yin, W.-G. Ma, R.-Y. Zhang and H.-S. Hou, AO Z0 associated production at the Large Hadron collider in the minimal supersymmetric standard model, Phys. Rev. D 66 (2002) 095008 [hep-ph/0209279] [INSPIRE].

[46] C. Kao, G. Lovelace and L.H. Orr, Detecting a Higgs pseudoscalar with a $Z$ boson at the LHC, Phys. Lett. B 567 (2003) 259 [hep-ph/0305028] [INSPIRE].

[47] C. Kao and S. Sachithanandam, Detecting a Higgs pseudoscalar with a $Z$ boson produced in bottom quark fusion, Phys. Lett. B 620 (2005) 80 [hep-ph/0411331] [INSPIRE].

[48] L.L. Yang, C.S. Li, J.J. Liu and L.G. Jin, Production of scalar Higgs bosons associated with Z0 boson at the CERN LHC in the MSSM, J. Phys. G 30 (2004) 1821 [hep-ph/0312179] [INSPIRE].

[49] Q. Li, C.S. Li, J.J. Liu, L.G. Jin and C.-P. Yuan, Next-to-leading order QCD predictions for AO Z0 associated production at the CERN Large Hadron Collider, Phys. Rev. D 72 (2005) 034032 [hep-ph/0501070] [INSPIRE].

[50] B.A. Kniehl and C.P. Palisoc, Associated production of $Z$ and neutral Higgs bosons at the CERN Large Hadron Collider, Phys. Rev. D 85 (2012) 075027 [arXiv:1112.1575] [INSPIRE].

[51] O. Brein, R.V. Harlander and T.J.E. Zirke, vh@nnlo - Higgs strahlung at hadron colliders, Comput. Phys. Commun. 184 (2013) 998 [arXiv:1210.5347] [INSPIRE].

[52] J.M. Butterworth, A.R. Davison, M. Rubin and G.P. Salam, Jet substructure as a new Higgs search channel at the LHC, Phys. Rev. Lett. 100 (2008) 242001 [arXiv:0802.2470] [INSPIRE].

[53] R. Hamberg, W. van Neerven and T. Matsuura, A Complete calculation of the order $\alpha_{s}^{2}$ correction to the Drell-Yan K factor, Nucl. Phys. B 359 (1991) 343 [Erratum ibid. B 644 (2002) 403-404] [INSPIRE].

[54] O. Brein, R. Harlander, M. Wiesemann and T. Zirke, Top-quark mediated effects in hadronic Higgs-strahlung, Eur. Phys. J. C 72 (2012) 1868 [arXiv:1111.0761] [INSPIRE].

[55] L. Altenkamp, S. Dittmaier, R.V. Harlander, H. Rzehak and T.J.E. Zirke, Gluon-induced Higgs-strahlung at next-to-leading order QCD, JHEP 02 (2013) 078 [arXiv:1211.5015] [INSPIRE].

[56] S. Dawson, Radiative corrections to Higgs boson production, Nucl. Phys. B 359 (1991) 283 [INSPIRE].

[57] A. Djouadi, M. Spira and P. Zerwas, Production of Higgs bosons in proton colliders: $Q C D$ corrections, Phys. Lett. B 264 (1991) 440 [INSPIRE].

[58] D. Graudenz, M. Spira and P. Zerwas, QCD corrections to Higgs boson production at proton proton colliders, Phys. Rev. Lett. 70 (1993) 1372 [INSPIRE].

[59] M. Spira, A. Djouadi, D. Graudenz and P. Zerwas, Higgs boson production at the LHC, Nucl. Phys. B 453 (1995) 17 [hep-ph/9504378] [INSPIRE].

[60] R.V. Harlander and W.B. Kilgore, Next-to-next-to-leading order Higgs production at hadron colliders, Phys. Rev. Lett. 88 (2002) 201801 [hep-ph/0201206] [INSPIRE].

[61] C. Anastasiou and K. Melnikov, Higgs boson production at hadron colliders in NNLO QCD, Nucl. Phys. B 646 (2002) 220 [hep-ph/0207004] [INSPIRE]. 
[62] V. Ravindran, J. Smith and W.L. van Neerven, NNLO corrections to the total cross-section for Higgs boson production in hadron hadron collisions, Nucl. Phys. B 665 (2003) 325 [hep-ph/0302135] [INSPIRE].

[63] D. Eriksson, J. Rathsman and O. Stål, 2HDMC: two-Higgs-doublet model calculator physics and manual, Comput. Phys. Commun. 181 (2010) 189 [arXiv:0902.0851] [inSPIRE].

[64] D. Eriksson, J. Rathsman and O. Stål, 2HDMC: two-Higgs-doublet model calculator, Comput. Phys. Commun. 181 (2010) 833 [InSPIRE].

[65] R. Harlander, M. Krämer and M. Schumacher, Bottom-quark associated Higgs-boson production: reconciling the four- and five-flavour scheme approach, arXiv:1112.3478 [INSPIRE].

[66] T. Hahn, Generating Feynman diagrams and amplitudes with FeynArts 3, Comput. Phys. Commun. 140 (2001) 418 [hep-ph/0012260] [INSPIRE].

[67] T. Hahn and M. Pérez-Victoria, Automatized one loop calculations in four-dimensions and D-dimensions, Comput. Phys. Commun. 118 (1999) 153 [hep-ph/9807565] [InSPIRE].

[68] T. Hahn, CUBA: a library for multidimensional numerical integration, Comput. Phys. Commun. 168 (2005) 78 [hep-ph/0404043] [INSPIRE].

[69] O. Brein et al., Precision calculations for associated WH and ZH production at hadron colliders, hep-ph/0402003 [INSPIRE].

[70] LHC Higgs Cross Section Working Group collaboration, S. Dittmaier et al., Handbook of LHC Higgs Cross Sections: 1. Inclusive observables, arXiv:1101.0593 [INSPIRE].

[71] A. Martin, W. Stirling, R. Thorne and G. Watt, Parton distributions for the LHC, Eur. Phys. J. C 63 (2009) 189 [arXiv:0901.0002] [inSPIRE].

[72] H.-L. Lai et al., New parton distributions for collider physics, Phys. Rev. D 82 (2010) 074024 [arXiv: 1007.2241] [INSPIRE].

[73] R.D. Ball et al., Parton distributions with LHC data, Nucl. Phys. B 867 (2013) 244 [arXiv:1207.1303] [INSPIRE].

[74] S. Biswas, E. Gabrielli and B. Mele, Single top and Higgs associated production as a probe of the Ht $\bar{t}$ coupling sign at the LHC, JHEP 01 (2013) 088 [arXiv:1211.0499] [INSPIRE].

[75] S. Biswas, E. Gabrielli, F. Margaroli and B. Mele, Direct constraints on the top-Higgs coupling from the $8 \mathrm{TeV}$ LHC data, JHEP 07 (2013) 073 [arXiv:1304.1822] [INSPIRE].

[76] C. Englert, M. McCullough and M. Spannowsky, Gluon-initiated associated production boosts Higgs physics, arXiv:1310.4828 [INSPIRE].

[77] G. Ferrera, private communication.

[78] R.V. Harlander, S. Liebler and H. Mantler, SusHi: a program for the calculation of Higgs production in gluon fusion and bottom-quark annihilation in the Standard Model and the MSSM, Comput. Phys. Commun. 184 (2013) 1605 [arXiv:1212.3249] [INSPIRE]. 